\title{
基于间苯三酚及其衍生物构筑新型有机多孔材料
}

\author{
邓汉林 ${ }^{a}$ 罗贤升 ${ }^{a}$ 李志华 ${ }^{b}$ 赵江颖 ${ }^{b}$ 黄木华* ${ }^{a}$ \\ $\left({ }^{a}\right.$ 北京理工大学材料学院 北京 100081) \\ ${ }^{b}$ 甘肃省银光化学工业集团有限公司 甘肃白银 730900)
}

\begin{abstract}
摘要 有机多孔材料凭借其高比表面积、孔道可调性、易功能化修饰和结构多样性等特征, 在催化、能源、吸附与分 离等多个领域中展现了巨大应用潜力. 对多官能度有机化合物单体以及高效聚合反应的巧妙应用, 为新型有机多孔材 料的创制提供了强有力的工具, 成为该领域的研究热点. 以 $C_{3}$ 对称型的间苯三酚及其衍生物为例, 综述了其在一系列 新型有机多孔材料高效构筑中的最新研究进展.
\end{abstract}

关键词 间苯三酚; 多孔高分子材料; 金属有机框架; 共价有机框架; 功能化

\section{Synthesis of Novel Porous Organic Materials Based on Phloroglucinol and Its Derivatives}

\author{
Deng, Hanlin ${ }^{a} \quad$ Luo, Xiansheng $^{a} \quad$ Li, Zhihua $^{b} \quad$ Zhao, Jiangying $^{b} \quad$ Huang, Muhua ${ }^{*, a}$ \\ ( ${ }^{a}$ School of Materials Science and Engineering, Beijing Institute of Technology, Beijing 100081) \\ ( ${ }^{b}$ Gansu Yinguang Chemical Industry Group Co., Ltd, Baiyin, Gansu 730900)
}

\begin{abstract}
Porous organic materials have found many applications, such as catalysis, energy, adsorption and separation., owing to the high specific surface area, pore channel tunability, easy functionalization and structural diversity. The careful selection of specific monomers with polyfunctionality and highly efficient polymerization provides powerful tools for creating novel porous organic materials, and it has become a research topic in this field. Herein the latest progress in the efficient construction of novel porous organic materials is reviewed taking $C_{3}$ symmetrical phloroglucinol and its derivatives as examples.
\end{abstract}

Keywords phoroglucinol; porous organic polymer; metal organic framework; covalent organic framework; functionalization

间苯三酚是一种重要的化工原料与合成中间体, $C_{3}$ 对称型多羟基芳香结构赋予其优秀的化学转化能力, 在 医药化工 ${ }^{[1-3]}$ 、含能材料 ${ }^{[4-5]}$ 及能源材料 ${ }^{[6-7]}$ 等领域发挥着 重要作用(Scheme 1). 其中, 间苯三酚及其多种衍生物 (如黄酮类化合物) 已被用于抗艾滋病药物 ${ }^{[1]}$ 和抗肿瘤药 物 ${ }^{[2]}$ 等重要药物的开发研究中. 此外, 基于间苯三酚及 其衍生物所具有的优秀化学转化反应平台, 研究者们已 经开发了包括有机多孔材料在内的一系列先进材料.

从天然沸石直至近二十多年来兴起的介孔和大孔 材料, 乃至多级孔材料, 其有序的多孔骨架组成主要为 无机化合物. 直至近十多年来, 以金属有机杂化骨架材 料为主体构筑的有机多孔骨架(MOFs) 和纯有机化合物 构筑的有机多孔骨架 $(\mathrm{COFs})$ 大量兴起, 正在成为晶态多

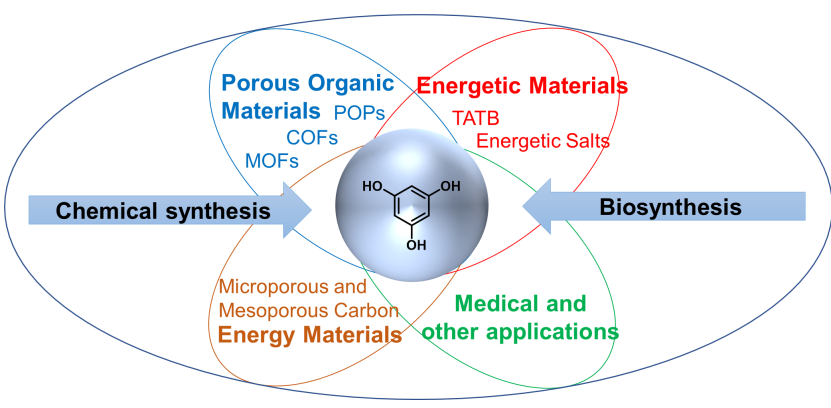

图式 1 间苯三酚的合成与应用

Scheme 1 Synthesis and applications of phloroglucinol

孔材料领域的前沿研究中心. 此外, 以工程应用为导向 的多孔材料要求充分考虑化学稳定性、制备实用性、实

* Corresponding author. E-mail: mhhuang@bit.edu.cn

Received May 26, 2020; revised July 9, 2020; published online September 8, 2020.

Project supported by the National Natural Science Foundation of China (No. 21772013) and the Natural Science Foundation of Beijing (No. 2202049). 国家自然科学基金(No. 21772013)和北京市自然科学基金(No. 2202049)资助项目. 
际应用效果和经济成本等因素. 因此, 超交联聚合物 $(\mathrm{HCPs})^{[8-10]}$ 、共轭微孔聚合物(CMPs $)^{[11-13]}$ 、固有微孔聚 合物(PIMs) ${ }^{[14-15]}$ 和多孔芳香骨架(PAFs) ${ }^{[16-18]}$ 等非晶态多 孔高分子材料也受到人们的高度关注. 对于上述有机多 孔材料的结构设计, 关键是选择具有多反应位点和一定 拓扑结构的有机化合物单体以及高效率的聚合反应. 近 年来, 许多优秀的多官能度有机化合物单体以及高效的 聚合反应 ${ }^{[19-22]}$ 被巧妙地应用于有机多孔材料的构筑中. 本综述选取具有 $C_{3}$ 对称性的间苯三酚为典型例子, 概 括总结了间苯三酚及其衍生物在构筑晶态与非晶态有 机多孔材料中的应用研究进展.

\section{1 间苯三酚在晶态有机多孔材料中的应用研究}

晶态有机多孔材料是具有较高结晶度的结构高度 规整的有机多孔材料, 包括共价有机框架 $(\mathrm{COFs})$ 与金属 有机框架 $(\mathrm{MOFs})^{[23-24]}$ 等. 这些晶态框架材料由于其可 调节的高度规整的结构、高结晶度及高比表面积等特性 而具有理论研究意义与实用价值. 间苯三酚具有 $C_{3}$ 对 称型三羟基芳香体系, 可通过丰富的 $\mathrm{O}$-和 $\mathrm{C}$-功能化转 化等得到一系列结构多样的有机化合物, 为晶态有机多 孔材料的研究提供了优秀的配体或单体. 下面将分别从 MOFs 和 COFs 两方面介绍间苯三酚在晶态有机多孔材 料构筑中的应用进展情况.

\section{1 间苯三酚及其衍生物在 COFs 材料中的应用研究}

COFs 属于晶态的有机多孔高分子材料, 由小分子 单体以共价键键合形成. 其合成关键在于选取恰当的聚 合单体、聚合方法以及聚合条件, 使聚合过程中以可逆 共价键形式键接单体, 并发生 “聚合(无序)-解聚-聚合 (有序)” 的过程, 从而获得高结晶度的 COFs 材料. 目前 主流的合成方法包括 Schiff base 生成反应、酮胺缩聚反 应、酰亚胺生成反应、硼酸酯化反应、硼酸自缩聚反应、 氭醛缩聚、氰基自缩聚反应、苯并噁唑生成反应等 ${ }^{[25]}$, 涉及聚合单体包括多元的胺类、醛类、酸䣶、酮类、酚 类、嗍酸及腈类等. 虽然间苯三酚 $C_{3}$ 对称型的羟基分布 使其难以参与硼酸酯化等需要邻位羟基的反应, 但其优 良的可修饰性能使其具有通过化学衍生策略得到高性 能单体的应用潜力, 基于间苯三酚衍生得到单体以合成 新型 COFs 材料的研究因此受到瞩目. 以下将分别介绍 间苯三酚及其不同衍生物在 COFs 材料中的应用研究情 况.

1.1.1 基于间苯三酚衍生的三甲醛基间苯三酚(TFP) 构筑化学稳定的 COFs 材料

间苯三酚醛类衍生单体以三甲醛基间苯三酚(TFP, 1)受到的关注最多, 利用 Duff 反应引入的醛基赋予了间 苯三酚参与酮胺缩合等反应的能力, 从而大大提升了间
苯三酚构筑 COFs 材料的应用性能. 2012 年, Banerjee 等 ${ }^{[26]}$ 将可逆的亚胺键与不可逆的烯胺键相结合, 巧妙 地在合成过程中保留可逆共价键动态调整特性, 形成结 晶框架后使其异构形成不可逆共价键, 从而制备了化学 稳定的 COFs 材料 TpPa-1 和 TpPa-2 (Scheme 2), 其最大 BET 比表面积为 $535 \mathrm{~m}^{2} \cdot \mathrm{g}^{-1}$, 并在强酸、强碱及高温水 浴等条件下仍能稳定保持高结晶度与高比表面积特性. 随后 Banerjee 等继续利用 TFP 构筑了一系列新型 COFs, 并基于这些材料研究了 $\mathrm{COFs}$ 材料的新型合成方法以及 其在催化、吸附等方面的应用 ${ }^{[27-30]}$. TFP 制备 COFs 材料 过程中的 “可逆一不可逆” 转化形成稳定化学结构同样 吸引了其他研究者们的关注. 2013 年, Dichtel 等 $^{[31]}$ 以 TFP 分别与对苯二胺、 2,6 -二氨基葱醌缩聚得到二维结 构的 DAB-TFP COF 和 DAAQ-TFP COF (Scheme 2), 二 者最大 BET 比表面积分别为 365 和 $1800 \mathrm{~m}^{2} \cdot \mathrm{g}^{-1}$, 并具 备作为稳定赝电容的应用潜力, 在 5000 次充放电循环 后保持较低的容量损耗, 他们随后继续跟进研究基于 TFP 构筑的酮胺键接的二维 COFs 在能源储存方面的应 用 ${ }^{[32-34]}$. 刘晓明课题组 ${ }^{[35]}$ 以 TFP 与水合肼构筑了二维多 孔结构的 COF-JLU2 (Scheme 2), 其高比表面积 $\left(S_{\mathrm{BET}}=\right.$ $415 \mathrm{~m}^{2} \cdot \mathrm{g}^{-1}$ )、规整的微孔结构(以 $0.96 \mathrm{~nm}$ 的微孔结构为 主)和丰富的杂原子基团赋予了材料优良的气体吸附性 能, 在 $273 \mathrm{~K}, 100 \mathrm{kPa}$ 条件下对 $\mathrm{CO}_{2}$ 和 $\mathrm{CH}_{4}$ 的吸附量分 别为 4.93 和 $2.38 \mathrm{mmol} \cdot \mathrm{g}^{-1}$. 随后姜忠义课题组以 TFP 与水合肼缩聚构筑了二维多孔 $\mathrm{TpHZ}$ 纳米片, 并使其同 聚醚砜复合成膜，在水/乙醇混合体系分离中实现 2.48 $\mathrm{kg} \cdot \mathrm{m}^{-2} \cdot \mathrm{h}^{-1}$ 的膜通量以及高分离常数 $(1430)^{[36]}$, 并持续 跟进了基于 TFP 构筑的 COF 纳米片在复合膜方面的应 用 ${ }^{[37-39]}$. TFP 单体的出现推动了高化学稳定性 COFs 材 料的发展, 进一步丰富了 COFs 结构, 对于 COFs 材料研 究发展有着重要意义.

\subsection{2 基于间苯三酚衍生的多氨基苯构筑 $\mathrm{COFs}$ 和氮 化碳材料}

间苯三酚的胺类衍生物单体包括三氨基间苯三酚 (TAPG, 2)与六氨基苯(HAB, 3)等, 其氨基官能团比羟基 官能团具有更加丰富多彩的转化化学，已经被用于构筑 结构新颖的 COFs 材料. TAPG 最初被用作三氨基间三羟 基环已烷的合成前体, 后者由于具备与金属离子极强的 螯合作用而被用作工业与医药螯合剂 ${ }^{[40]}$. 但羟基氨基 交错对称排布的结构仍然吸引了材料领域研究者的兴 趣. 2019 年, Baek 等 ${ }^{[41]}$ 以 TAPG 与对苯二甲醛聚合, 制 备了亚胺类的 I-COF (Scheme 3), 并借助 “可逆一不可 逆” 共价键的转化, 进一步得到了高化学稳定性的啞唑 环结构的 BO-COF (Scheme 3), 二者 BET 比表面积分别 为 1906 和 $1687 \mathrm{~m}^{2} \cdot \mathrm{g}^{-1}$. 目前关于 TAPG 在 COFs 材料 

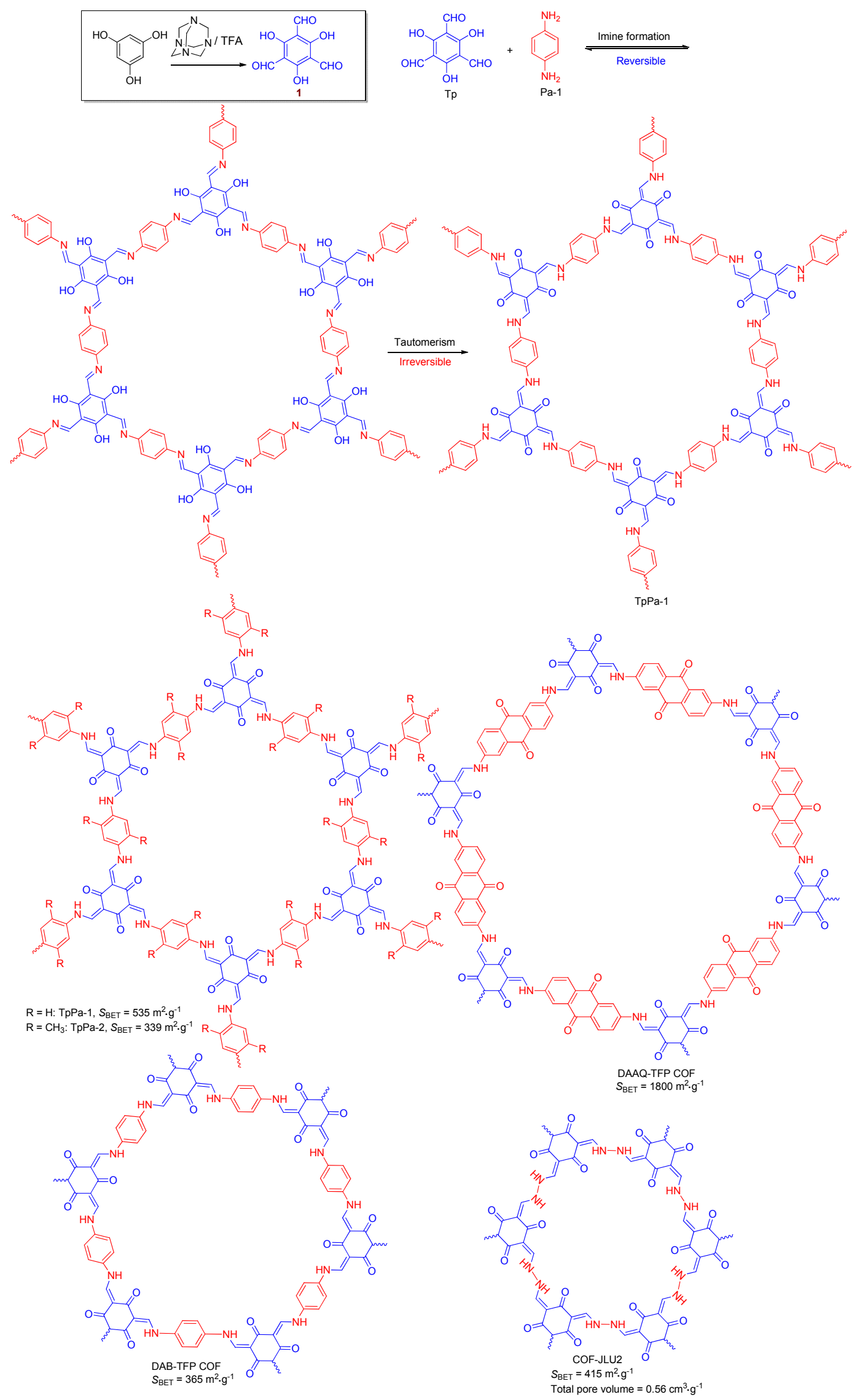

图式 2 基于 TFP 发展的稳定化 COFs 材料

Scheme 2 Developing stable COFs based on TFP 

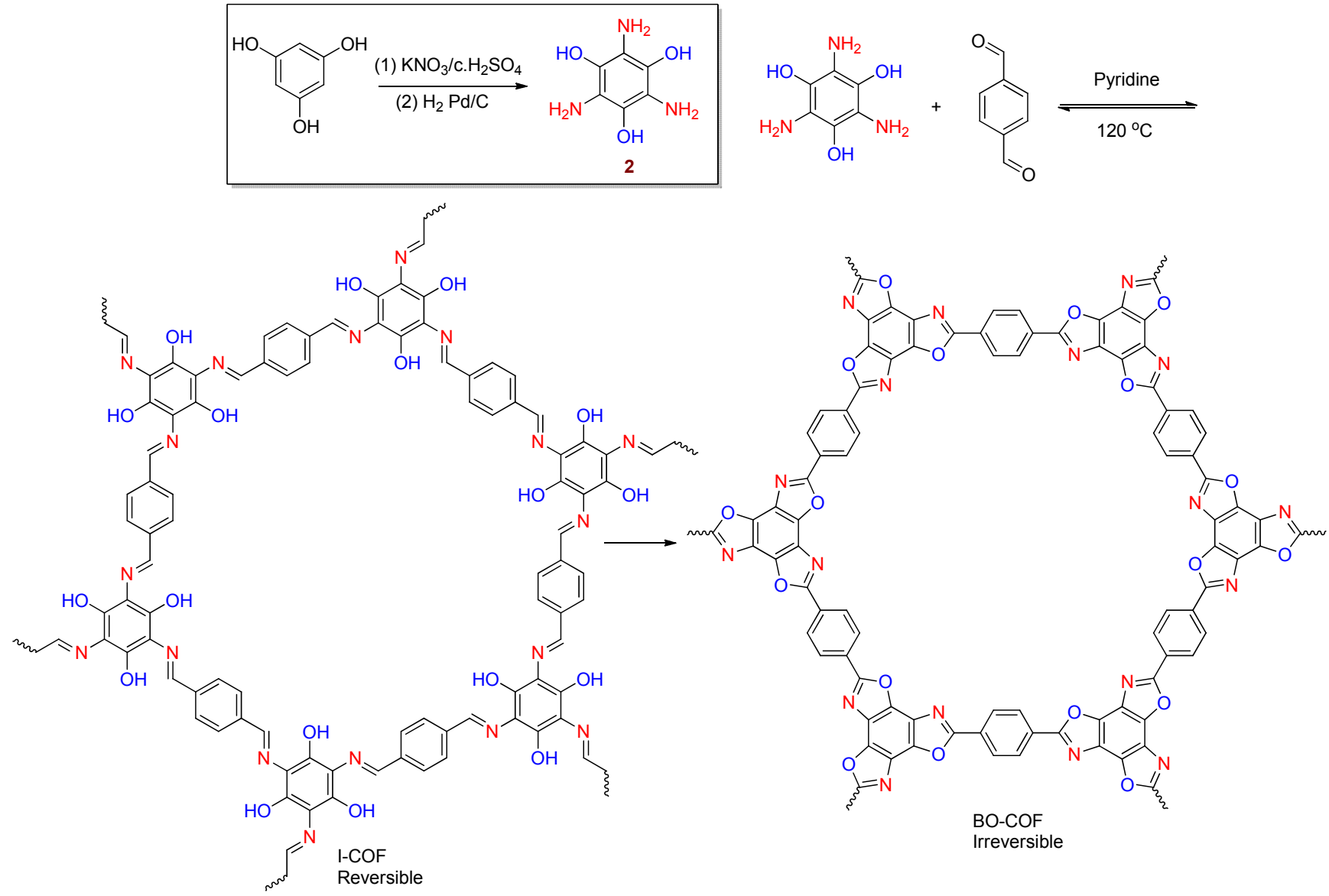

图式 3 基于 TAPG 的稳定的 COFs 材料

Scheme 3 Developing COFs based on TAPG

中的应用研究仍处于起步阶段, 利用羟基与氨基的相关 化学反应还能构筑其它化学结构键接的 COFs 材料, 基 于 TAPG 的新型高化学稳定性 COFs 材料的合成应用还 有待进一步研究.

$\mathrm{HAB}$ 结构中的六个氨基给苯环供电子, 使其可作 为三个邻苯二胺参与化学反应, 具有极高的化学反应活 性. 2015 年, Baek 等 ${ }^{[22]}$ 报道了基于 $\mathrm{HAB}$ 与环己六酮缩聚 制备类石墨烯结构的新型晶态多孔氮化碳材料 $\mathrm{C}_{2} \mathrm{~N}$ (Scheme 4, a). 不同于石墨烯的连续六元环蜂窝结构, $\mathrm{C}_{2} \mathrm{~N}$ 具有均匀 $\mathrm{N}$ 原子分布的六元环围绕的孔洞结构, 从 而具备良好的半导体性能, 其带隙为 $1.96 \mathrm{eV}$, 基于 $\mathrm{C}_{2} \mathrm{~N}$ 的场效应晶体管表现出高达 $10^{7}$ 的开关比; 同时其多氮 芳环网络结构赋予 $\mathrm{C}_{2} \mathrm{~N}$ 在催化、能源、气体吸附与分离、 生物应用等领域中应用的巨大潜力. Baek 等陆续以 $\mathrm{C}_{2} \mathrm{~N}$ 负载 $\mathrm{Co}^{[43]} 、 \mathrm{Ru}^{[44]} 、 \mathrm{Fe}^{[45]}$ 制备了负载型金属催化剂, 其 在析氢反应(HER)和氧还原反应(ORR)中表现出优秀的 催化应用性能. 但 HAB 传统的合成方法是含能材料 1,3,5-三氨基-2,4,6-三硝基苯(TATB)还原法, 往往需要 研究者自行合成 TATB, 增加了合成的危险因素和难度. 因此解决 $\mathrm{HAB}$ 单体的合成难题或是另辟蹊径拓展 $\mathrm{C}_{2} \mathrm{~N}$
的新型合成方法均具有重要意义. 2018 年, Oschatz 等 ${ }^{[46]}$ 以六氧基氮杂芳香单体(HAT)在高温条件下实现氰基间 的固相缩聚制得 $\mathrm{C}_{2} \mathrm{~N}$ (Scheme $4, \mathrm{~b}$ ); 同年, Lee 等 ${ }^{\left[{ }^{[7]} \text { 则从 }\right.}$ 氯冉酸出发, 经胺化、聚合及还原步骤, 引入 $\mathrm{S}$ 原子构 建了三维 $\mathrm{S}-\mathrm{C}_{2} \mathrm{~N}$ 气凝胶材料, 该气凝胶表现出了优秀的 电化学应用性能 (Scheme 4, c). 以上方法均降低了 $\mathrm{C}_{2} \mathrm{~N}$ 的合成难度, 为 $\mathrm{C}_{2} \mathrm{~N}$ 的应用及深入研究提供了良好基 础.

\subsection{3 基于其它间苯三酚衍生物构筑 COFs 材料}

基于间苯三酚的 $\mathrm{O}$-功能化反应和 $\mathrm{C}$-功能化反应等 衍生策略, 目前已得到大量间苯三酚衍生物单体, 并且 这些单体被成功应用于新型 COFs 材料构筑之中. 冯守 华课题组 ${ }^{[48]}$ 以间苯三酚与三聚氰氯构筑了氧杂杯芳烃 类单体 $\mathbf{4}$, 并将其与四(4-氨基苯基)甲烷聚合得到了高 稳定的荧光 COFs 材料 COF-TT (Scheme 5), 其 BET 比 表面积为 $446 \mathrm{~m}^{2} \cdot \mathrm{g}^{-1}$, 其丰富的杂原子增大了材料与离 子间的相互作用, 能够用于溶液中 $\mathrm{Fe}^{3+} 、 \mathrm{CrO}_{4}^{2-}$ 、 $\mathrm{Cr}_{2} \mathrm{O}_{7}^{2-}$ 和 $\mathrm{MnO}_{4}^{-}$离子的探测, 并在 $\mathrm{pH} 2 \sim 14$ 的环境中 均能保持结构的完整性. 
(a)<smiles>Nc1c(N)c(N)c(N)c(N)c1N</smiles>

3<smiles>N#Cc1nc2c3nc(C#N)c(C#N)nc3c3nc(C#N)c(C#N)nc3c2nc1C#N</smiles>

Polymerization<smiles>O=C1C(=O)C(=O)C(=O)C(=O)C1=O</smiles>

(c)<smiles>Nc1c(N)c(N)c(N)c(N)c1N</smiles>

图式 $4 \mathrm{C}_{2} \mathrm{~N}$ 的合成

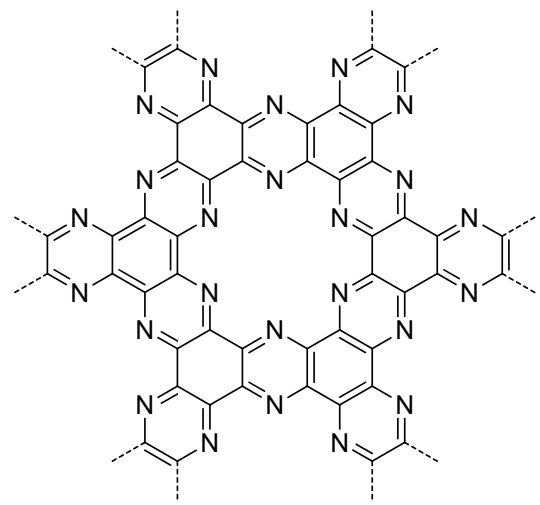

Polymerization

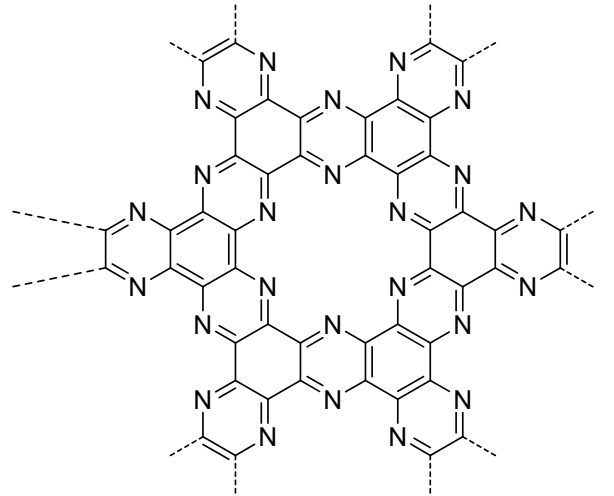

L-cysteine

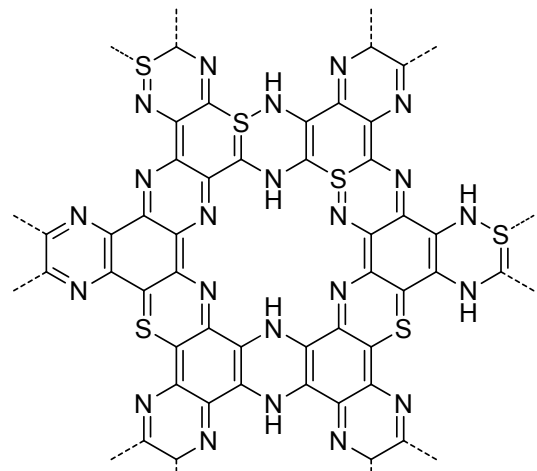

Scheme 4 Synthesis of $\mathrm{C}_{2} \mathrm{~N}$

而王其强课题组 ${ }^{[49]}$ 利用间苯三酚衍生的三维笼状 分子结 5 作为多醛类单体, 将其与对苯二胺、 4,4'-二氨 基联苯等进行缩聚, 合成了新型笼状 COFs 材料 cageCOF-1 和 cage-COF-2 (Scheme 6), 其 BET 比表面积分别 为 1237 和 $667 \mathrm{~m}^{2} \cdot \mathrm{g}^{-1}$. 该工作进一步增加了三维 COFs 的结构复杂度, 为笼状 COFs 的设计合成提供了新的思 路.

张振杰课题组 ${ }^{[50]}$ 以间苯三酚的偶氮衍生物 6 作为 关键单体, 与二官能度的 2,5 -二羟基对苯二甲醛以及三 官能度的 TFP、1,3,5-三(4-甲酰基苯基)苯和 1,3,5-三(3羟基-4-甲酰基苯乙炔苯基)苯分别聚合得到 NKCOF-2, NKCOF-1, NKCOF-3, NKCOF-4 (Scheme 7), 其 BET 比 表面积分别为 $1510,1011,1139$ 和 $2612 \mathrm{~m}^{2} \cdot \mathrm{g}^{-1}$, 孔径分 布集中于 $3.3,1.8,2.4$ 和 $2.8 \mathrm{~nm}$, 具有优秀的多孔性能.
其丰富的偶氮键和羟基等活性基团的存在更赋予了 COFs 独特的性能. 利用偶氮基团对磷酸的强结合作用 负载磷酸 $\left(\mathrm{H}_{3} \mathrm{PO}_{4}\right)$ 后, $\mathrm{H}_{3} \mathrm{PO}_{4} @ \mathrm{NKCOF}-1$ 电导率达 $1.13 \times$ $10^{-1} \mathrm{~S} \cdot \mathrm{cm}^{-1}(353 \mathrm{~K}, 98 \%$ 相对湿度条件下), 为目前 COFs 材料的最高记录. 此外, $\mathrm{H}_{3} \mathrm{PO}_{4} @ \mathrm{NKCOF}-1$ 膜还表 现出 $81 \mathrm{~mW} \mathrm{~cm}{ }^{-2}$ 的功率密度和 $456 \mathrm{~mA} \cdot \mathrm{cm}^{-2}$ 的最大电 流密度, 并在强酸碱环境或沸水中仍能保持高度的稳定 性，在燃料电池领域中具有可观的应用前景.

上述研究充分展示了间苯三酚及其衍生物在 COFs 材料构筑研究中的巨大鬼力. 间苯三酚可以基于 O-功 能化反应得到复杂结构的单体构筑 COFs 材料, 或通过 C-功能化反应引入多醛基、多氨基官能团后与胺、醛缩 合构筑 COFs 材料. 其中, 在基于多醛基衍生单体 TFP 的 COFs 材料中, 羟基的存在帮助不稳定的亚胺实现不 


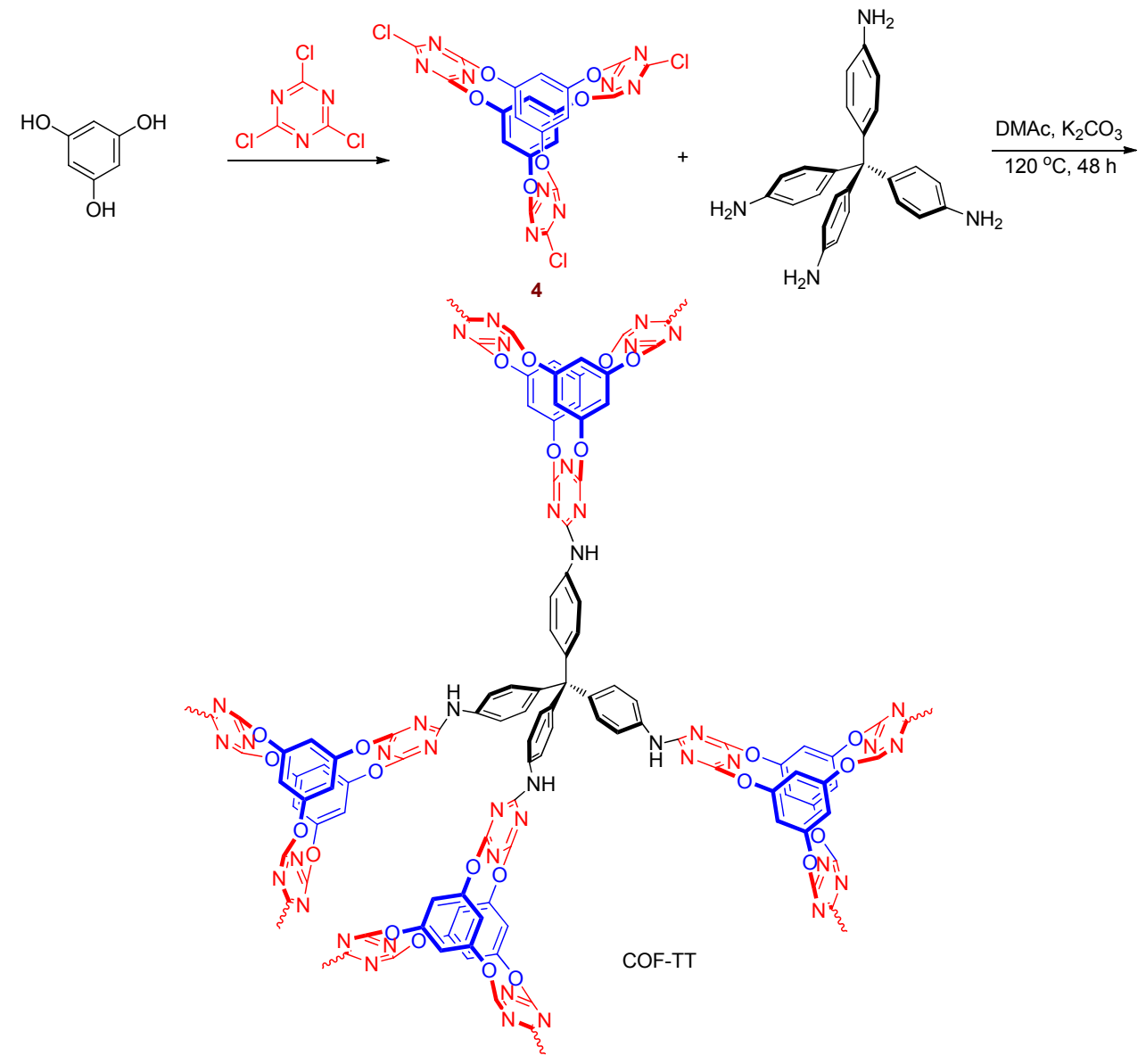

图式 5 COF-TT 的合成

Scheme 5 Synthesis of COF-TT

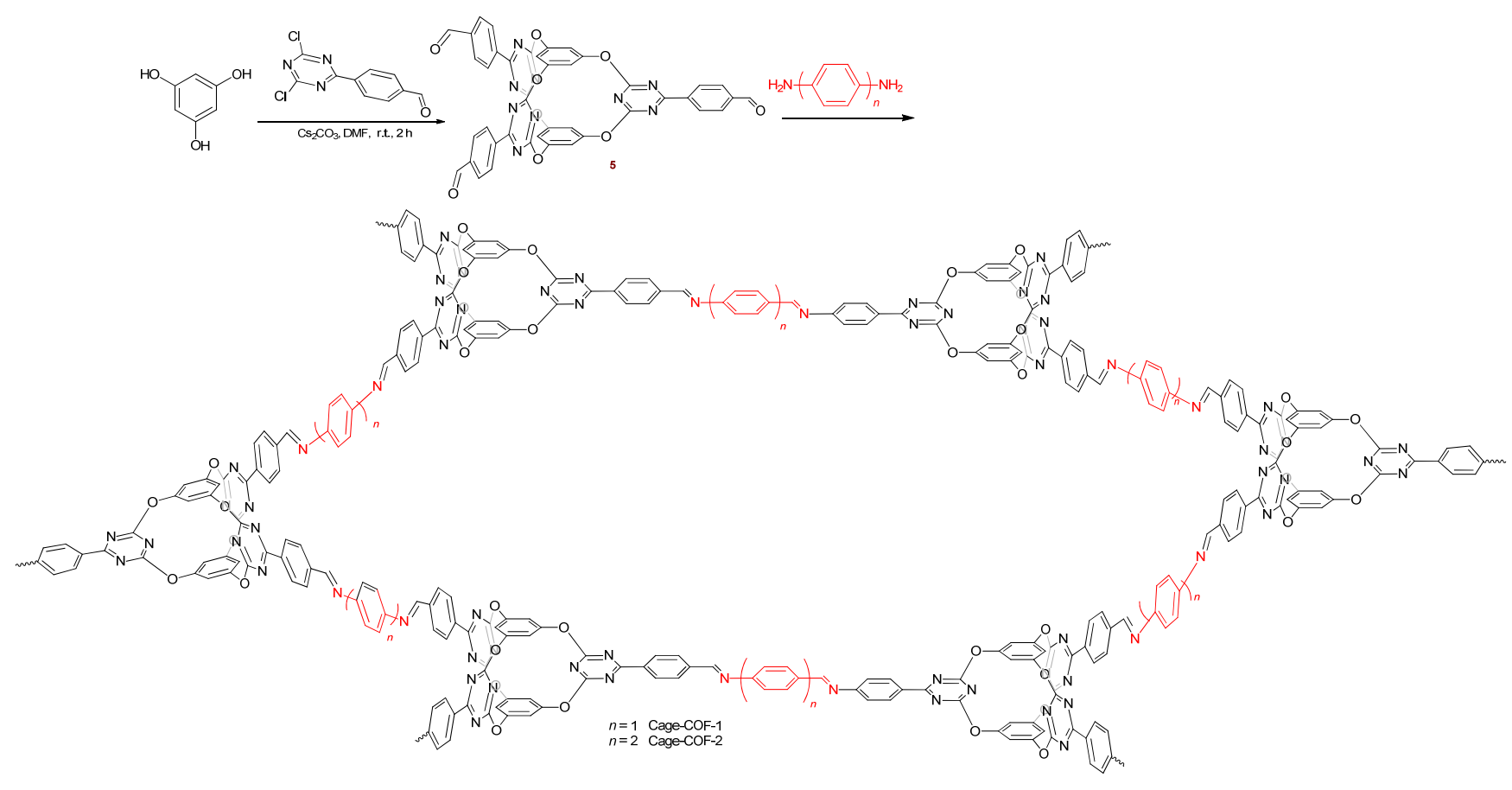

图式 6 Cage-COFs 的合成

Scheme 6 Synthesis of cage-COFs 


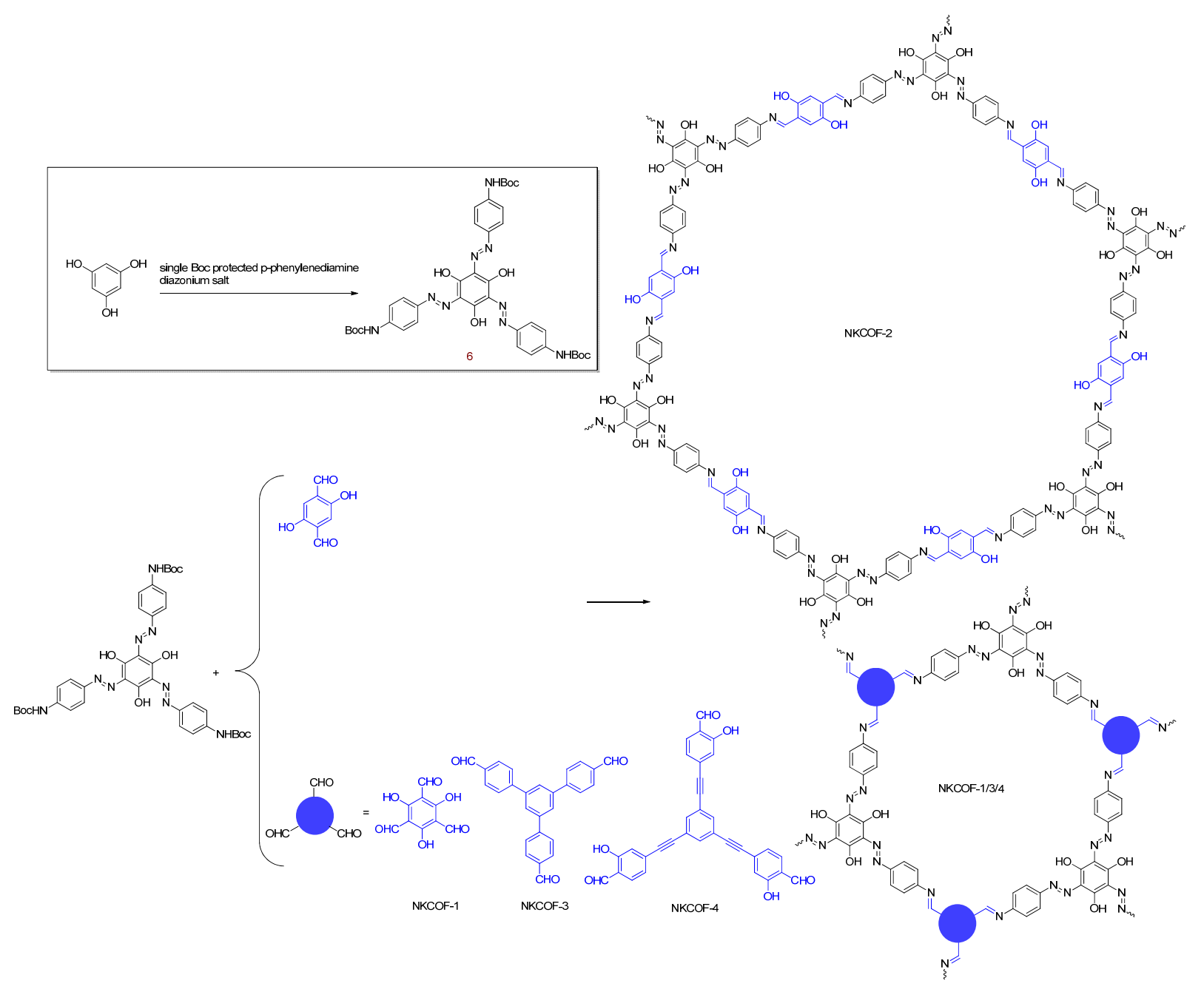

图式 7 Cage-COFs 结构

Scheme 7 Structures of cage-COFs

可逆异构形成了稳定的烯胺结构 ${ }^{[26]}$, 从而大大提升了 COFs 材料的化学稳定性; 在基于多氨基衍生单体 TAPG 的 COFs 材料中, 邻位羟基与亚胺进一步反应形 成稳定的噁唑环结构 ${ }^{[41]}$, 同样使 COFs 材料获得了更高 的化学稳定性. 此外, 基于间苯三酚的多氨基衍生单体 $\mathrm{HAB}$ 构筑的氮化碳材料, 已开始崭露头角, 并将在氮化 碳及新型 $\mathrm{COFs}$ 材料中扮演越来越重要的角色.

而除了化学结构上的设计, 间苯三酚及其衍生物对 于 $\mathrm{COFs}$ 材料的拓扑结构的设计同样有着重要作用. 一 般而言, 具有 $C_{3} 、 C_{6}$ 对称性的间苯三酚及其衍生物所构 筑的二维 COFs 材料往往呈六方结构. 如具有 $C_{3}$ 对称性 的间苯三酚及其衍生物与 $C_{2} 、 C_{3}$ 型单体连接构筑的二 维六方结构的 COFs 材料、基于 TFP 与二元胺构筑的多 种 COFs 材料和张振杰课题组的 NKCOFs 材料 ${ }^{[50]}$ 即是此 类; 如具有 $C_{6}$ 对称性的间苯三酚及其衍生物与另一 $C_{6}$ 单体构筑二维六方结构的 COFs 材料, 六氨基苯构筑的 氮化碳类 $\mathrm{COFs}$ 材料 $\mathrm{C}_{2} \mathrm{~N}^{[42]}$ 即属此列. 而此类 $C_{6}$ 型单
体还可与 $C_{2}$ 等对称型单体连接构筑具有三角孔道等不 同拓扑结构的新型 COFs 材料, 这方面的工作有待更多 的研究. 而除了二维六方 $\mathrm{COFs}$ 材料以外, 具有 $C_{3}$ 对称 性的间苯三酚及其衍生物还能与 $T_{4}$ 型连接单体连接构 筑具有立体结构的三维 COFs 材料, 如冯守华课题组 ${ }^{[48]}$ 构筑的 COF-TT 材料. 在三维 COFs 方兴未艾的今天, 利用 $C_{3} 、 C_{6}$ 对称性的间苯三酚及其衍生物通过巧妙的 化学与拓扑设计合成新型的三维 COFs 材料仍有巨大的 研究价值. 因此未来间苯三酚的醛类、多氨基类等对称 衍生物将在新型高性能 COFs 材料构筑中展现出巨大的 应用价值.

\section{2 间苯三酚及其衍生物在 MOFs 材料中的应用研究}

MOFs 材料领域的蓬勃发展受益于丰富多样的优秀 配体. 虽然间苯三酚受限于自身结构一般难以直接与金 属离子键接形成稳定的框架结构, 但间苯三酚的衍生物 在新型 MOFs 材料的合成中受到较多关注.

1999 年, Abrahams 等 ${ }^{[51]}$ 以间苯三酚衍生的 2,4,6-三 
偶氮苯基-1,3,5-三羟基苯(tapp，7)作配体构筑了笼状大 分子 $\mathrm{Cu}_{12}(\operatorname{tapp})_{8}(\mathrm{Scheme} 8, \mathrm{a}) .2009$ 年, Hurd 等 ${ }^{[52]}$ 尝试 对间苯三酚进行磺酸化修饰, 得到了 2,4,6-三差基$1,3,5$-三苯磺酸盐配体 $(\mathbf{L} 1, \mathbf{8})$, 并选择 $\mathrm{Na}^{+}$与之配位得到 $\beta$-PCMOF2 (Scheme 8, b). 当其孔道中填充客体分子 $1 H-1,2,4-$ 三唑(Tz)后，在框架与客体分子的协同作用下， 其在 $150{ }^{\circ} \mathrm{C}$ 干燥氢气气氛中电导率达 $5 \times 10^{-4} \mathrm{~S} \cdot \mathrm{cm}^{-1}$; 而通过配体与客体分子的调控策略能够有效调控 PCMOF 的高温质子传导性能. 在此工作基础上, Kim 等 ${ }^{[53]}$ 采取同构配体置换策略, 以 2,4,6-三羟基- $1,3,5$-三苯 磺酸盐构筑 $\beta$-PCMOF2 后, 用 $1,3,5$-苯三膦酸进行配体 置换得到质子传导性能更优秀的 PCMOF, 电导率达 2.1 $\times 10^{-2} \mathrm{~S} \cdot \mathrm{cm}^{-1}\left(85{ }^{\circ} \mathrm{C}, 90 \%\right.$ 相对湿度条件下). 随后他们 更进一步提出同构配体置换与杂环掺杂协同配合策略, 在 $\beta$-PCMOF2 基础上衍生得到质子传导性能进一步提 高的 PCMOF21/2(Pz) 和 PCMOF21/2(Tz), 其电导率可达 $10^{-1} \mathrm{~S} \cdot \mathrm{cm}^{-1}$ 以上 $\left(85{ }^{\circ} \mathrm{C}, 90 \%\right.$ 相对湿度条件下). 2,4,6-三 羟基-1,3,5-三苯磺酸盐对于质子传导 MOFs 材料的研究 具有重要意义. 此外, 苏成勇课题组 ${ }^{[54]}$ 以间苯三酚与异 烟酰氯反应制备了骨架拓展的 1,3,5-三(异烟酸)苯配体
$(\mathrm{BTTP} 4,9)$, 并将其分别与 $\mathrm{CuI}$ 和 $\mathrm{CuBr}$ 配位构筑了两类 新型 MOFs 材料(Scheme 8, c), 其最大 BET 比表面积为 $496 \mathrm{~m}^{2} \cdot \mathrm{g}^{-1}$, 具有气体分离方面的应用价值. 李文军课 题组 ${ }^{[5]}$ 和 Chaudhari 等 ${ }^{[56]}$ 则分别以间苯三酚与 3-硝基邻 苯二甲腈和对氟苯甲腈合成了多羧基配体 $\mathrm{H}_{6} \mathrm{~L}(\mathbf{1 0})$ 和 $\mathrm{H}_{3} \mathrm{~L}$ (11), 并将配体分别同 $\mathrm{Zn}^{2+} 、 \mathrm{Cd}^{2+}$ 和 $\mathrm{Zn}^{2+}$ 配位制备 了新型 MOFs 材料(Scheme 8, d, e), 所得 MOFs 材料在 荧光性能上有突出表现, 前者对 $\mathrm{Fe}^{3+} 、 \mathrm{CrO}_{4}^{2-}, \mathrm{Cr}_{2} \mathrm{O}_{7}^{2-}$ 和 $\mathrm{MnO}_{4}^{-}$展示出极高的探测灵敏度, 后者对含硝基的 炸药化合物具有较高的探测能力. 间苯三酚还能用于 MOFs 材料的化学修饰. 2016 年, Rimoldi 等 ${ }^{[57]}$ 将间苯三 酚进行醚化修饰制备了钓配合物, 并将其接枝于 MOFs 材料 NU-1000 上，使之具有对烯烃的高效催化氢化性 能, 其对苯乙烯催化氢化的 TOF 值最高可达 $7.2 \mathrm{~h}^{-}$ ${ }^{1}$ (室温, $100 \mathrm{kPa}$ 条件下).

通过间苯三酚羟基的 $\mathrm{O}$-功能化反应引入羧基等官 能团，一方面扩大了配体骨架，另一方面引入了更多的 配位位点，协同作用不仅利于 MOFs 材料的构筑，还赋 予材料独特的性能, 如以多羧基配体 $\mathrm{H}_{6} \mathrm{~L}$ 和 $\mathrm{H}_{3} \mathrm{~L}$ 构筑得 到苂光 MOFs 材料(Scheme 8, d, e $)^{[55-56]}$. 通过间苯三酚

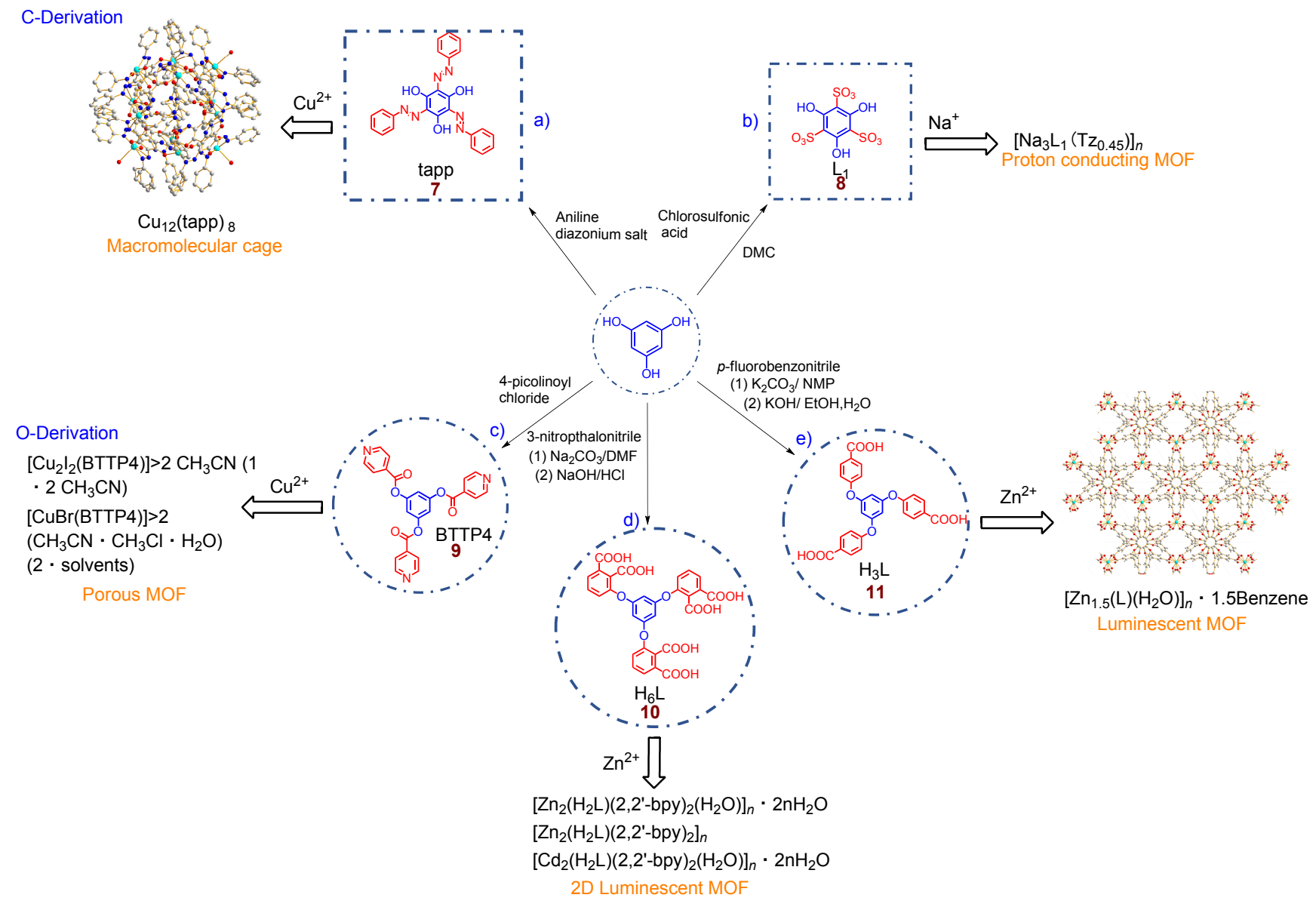

图式 8 基于间苯三酚衍生物的 MOFs 与配体

Scheme 8 MOFs and ligands based on derivatives of phloroglucinol 
$\mathrm{C}$-功能化反应引入官能团则注重于官能团与间苯三酚 骨架的协同作用, 如基于间苯三酚多磺酸功能化衍生物 构筑得到的高质子传导性的 PCMOF $^{[52]}$ (Scheme 8, b). 基于间苯三酚分子平台进行衍生化反应(O-功能化反应 和 C-功能化反应) 所得的衍生物在功能化 MOFs 材料中 展示出了可观的应用前景, 特别是间苯三酚的 O-功能 化反应获得的不同骨架和化学组成的衍生物, 能够实现 低成本制备多种具有不同晶体结构的高性能新型 MOFs 材料，间苯三酚及其衍生物在 MOFs 材料构筑中仍具有 巨大的潜力.

基于间苯三酚及其不同的功能化衍生反应(O-功能 化反应和 C-功能化反应), 一系列新型单体与配体被合 成出来并应用于 COFs、MOFs 材料的构筑当中, 并被进 一步的研究以揭示其对于高性能 COFs、MOFs 材料的 重要应用性能的贡献. 基于间苯三酚的 $\mathrm{O}$-功能化反应 可以实现分子骨架的扩张, 使之更适于构筑复杂框架结 构, 如 Cage- $\mathrm{COF}^{[49]}$ 和苂光 MOF 材料 ${ }^{[55-56]}$. 基于间苯三 酚的 C-功能化反应可以在保留多羟基体系的同时引入 其他官能团结构, 从而使单体/配体构筑的晶态有机多 孔材料具有独特的性能, 如 TFP 和 TAPG 构筑的多种高 化学稳定性 COFs 材料和 $\mathbf{L 1}$ 配体构筑的多种高质子传 导性的 MOFs 材料, 其中羰基、氨基和磺酸基团对于材 料性能有着极为重要的影响.

除了 COFs 和 MOFs 材料, 基于间苯三酚的功能化 衍生反应还能在晶态有机多孔分子笼(Porous Organic Cage)的构筑中发挥重要作用 ${ }^{[58]}$. 根据应用需求恰当地 选择功能化衍生反应策略, 间苯三酚及其衍生物在未来 不同种类的晶态有机多孔材料设计合成中仍将扮演重 要的角色.

\section{2 间苯三酚在非晶态多孔高分子材料构筑中的 应用研究}

相对于晶态的有机多孔材料, 非晶态的多孔高分子 材料的研究更加注重化学稳定性、合成方法的实用性以
及实际应用效果，同时更加依赖经典有机化学反应的巧 妙应用. 例如, McKoewn 课题组 ${ }^{[14]}$ 将芳香亲核取代反应 与构象扭曲单体巧妙结合，获得了可溶性的固有微孔高 分子(PIMs), Cooper 课题组 ${ }^{[12]}$ 借助 Suzuki 偶联和 Sonogashira 偶联反应等发展了共轭微孔高分子(CMPs), 朱广山和雸式纶课题组 ${ }^{[16]}$ 应用 Yamamoto 偶联聚合反应 制备了超高比表面积的 PAF-1, 谭必恩课题组 ${ }^{[9]}$ 利用经 典 Friedel-Crafts 和 Scholl 反应等实现了超交联高分子 ( $\mathrm{HCPs}$ ) 的实用制备, 韩宝航课题组 ${ }^{[59]}$ 利用咔唑的氧化 偶联反应制备了咔唑基多孔高分子材料 CPOPs，黄木华 课题组 ${ }^{[60-61]}$ 利用偶氮偶联聚合反应发展了偶氮类多孔 高分子材料 Azo-POPs. 得益于 $C_{3}$ 对称型三羟基芳香体 系的优秀反应平台，间苯三酚及其衍生物近年来被用于 多种聚合反应研究, 获得了一系列功能性多孔高分子材 料. 下面将从间苯三酚的 O-功能化反应、C-功能化反应 和间苯三酚衍生物的聚合反应三大合成策略分别介绍 目前间苯三酚在多孔高分子材料构筑中的应用进展.

\section{1 基于间苯三酚中羟基的 O-功能化反应策略构筑多 孔高分子}

理论上，间苯三酚的羟基可以直接发生 O-芳基化、 $\mathrm{O}$-酰化、O-醚化等功能化反应, 形成具有醚键、酯键和 苯并噁嗪环等化学结构键接的不同含 $\mathrm{O}$ 键接结构特征 的网状多孔高分子(Scheme 9). 基于该策略，借助羟基 的各种功能化反应，能实现制备 $\mathrm{O}$ 原子参与聚合键接的 不同结构的多孔高分子.

2013 年, 王忠刚课题组 ${ }^{[62]}$ 以间苯三酚的氧酸酯化 产物为单体自缩聚形成超交联网状聚氰腿酸酯 CE-P2 (表 1, Entry 1). 由于 1,3,5-三㲵酸酯苯单体具有较大的 位阻影响, 相比 1,3,5-三(4-氧基苯基)苯(聚合产物为 CE-P1, $S_{\mathrm{BET}}=630 \mathrm{~m}^{2} \cdot \mathrm{g}^{-1}$, 孔径分布集中于 0.57 和 1.01 $\mathrm{nm}$ )其聚合更加随机而难以得到规整微孔结构(孔径在 1.2 4.1 nm 间，呈现较宽分布)，但丰富的杂原子仍使 高分子表现出与 $\mathrm{CO}_{2}$ 较强的结合能力(在 $273 \mathrm{~K}, 100 \mathrm{kPa}$ 条件下吸附量为 $1.32 \mathrm{mmol} \cdot \mathrm{g}^{-1}$ ). Karmakar 和朱广山课

表 1 基于间苯三酚的 O-功能化反应构筑 POPs 总结

Table 1 Summary of POPs based on O-functionalized reaction

\begin{tabular}{clccc}
\hline Entry & Polymer & $S_{\mathrm{BET}} /\left(\mathrm{m}^{2} \cdot \mathrm{g}^{-1}\right)$ & Mean pore size $/ \mathrm{nm}^{-}$ & $V_{\text {tot }} /\left(\mathrm{g} \cdot \mathrm{cm}^{-3}\right)$ \\
\hline $1^{[62]}$ & CE-P2 & 195 & 3.39 & 0.198 \\
$2^{[63]}$ & CTF-IP10 & 358 & - & 0.22 \\
$3^{[65]}$ & COP-36 & 11 & - & 0.032 \\
$4^{[66]}$ & Si(OAc) $)_{4}$-based & 990 & 3.51 & 0.84 \\
$5^{[66]}$ & HSi(OAc) $)_{3}$-based & 933 & 5.99 & 1.36 \\
$6^{[66]}$ & MeSi(OAc) $)_{3}$-based & 455 & 2.73 & 0.285 \\
$7^{[67]}$ & BoxPOP-1 & 231 & - & 0.98 \\
$8^{[67]}$ & BoxPOP-2 & 225 & - & 0.74 \\
$9^{[67]}$ & BoxPOP-3 & 46 & - & 0.17 \\
$1^{[68]}$ & CPF-T & 654 & - & 0.33 \\
\hline
\end{tabular}




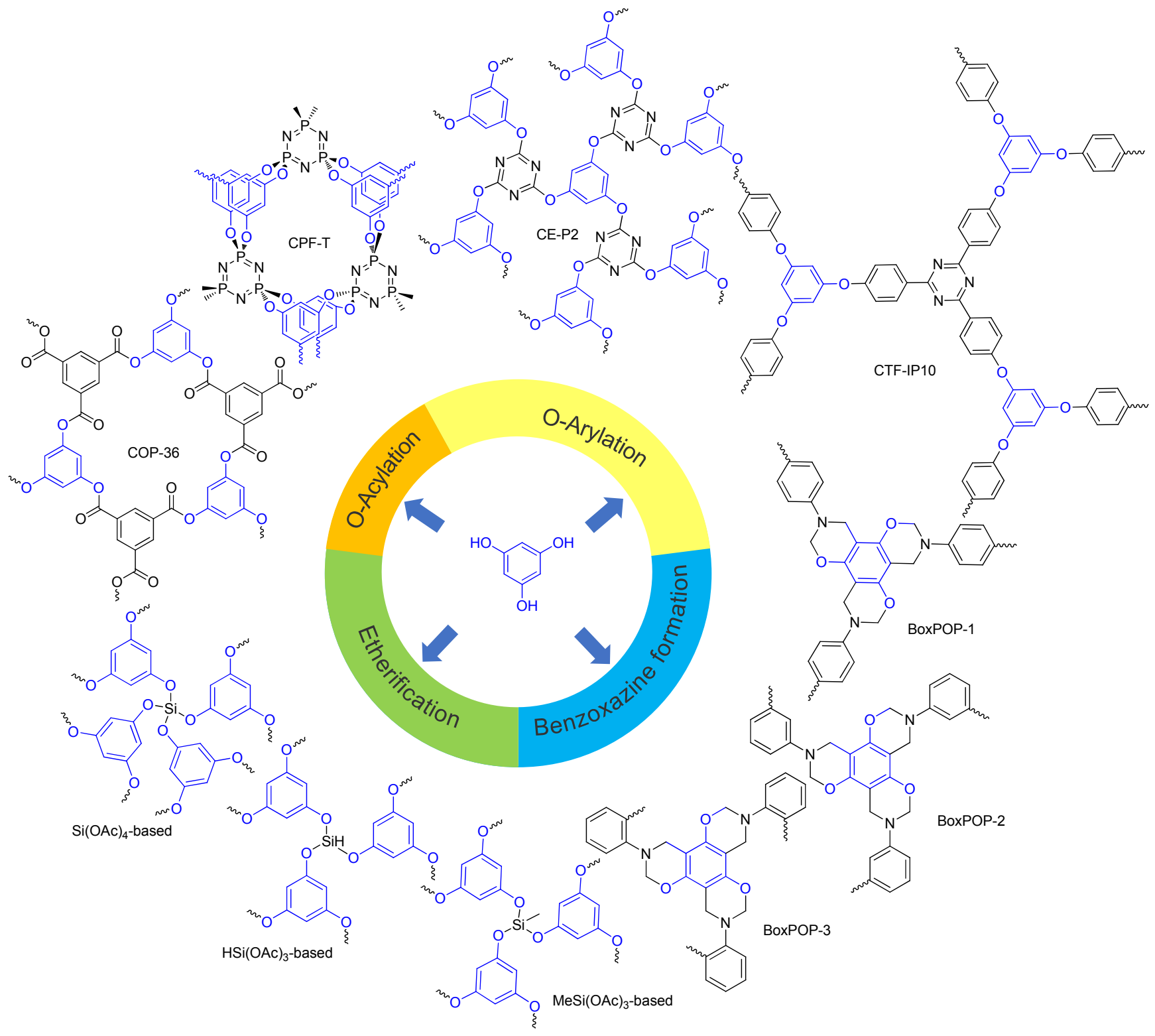

图式 9 基于间苯三酚 $\mathrm{O}$-功能化反应的 POPs

Scheme 9 POPs based on O-functionalized reactions

题组 ${ }^{[63]}$ 先后以对氟苯甲腈与间苯三酚缩合得到的 $1,3,5-$ 三(4-氰基苯氧基)-苯作为框架单体, 再于高温酸催化条 件下自缩聚得到共价三嗪框架 $(\mathrm{CTF})$ 和多孔芳香框架 (PAF), 前者 BET 比表面积 $358 \mathrm{~m}^{2} \cdot \mathrm{g}^{-1}$ (表 1 , Entry 2), 并 对爆炸物 2,4,6-三硝基苯酚具有极高的探测灵敏度; 后 者 Langmuir 比表面积为 $217 \mathrm{~m}^{2} \cdot \mathrm{g}^{-1}$, 以 $0.52 \mathrm{~nm}$ 孔径的 微孔为主, 在 $360 \mathrm{kPa}$ 压力下对 $\mathrm{CO}_{2}$ 和 $\mathrm{CH}_{4}$ 渗透速率分 别达到 $2.90 \times 10^{-7}$ 和 $1.29 \times 10^{-8} \mathrm{~mol} \cdot \mathrm{m}^{-2} \cdot \mathrm{s}^{-1} \cdot \mathrm{Pa}^{-1}$, 其 $\mathrm{CO}_{2} / \mathrm{CH}_{4}$ 渗透选择性达到 $22.48^{[64]} .2016$ 年, Ullah 等 ${ }^{[65]}$ 以 $1,3,5$-三苯甲酰氯为核心与包括间苯三酚在内的多个 芳香族二胺、二酚和三酚缩聚, 形成以酯键和酰胺键连 接的共价有机高分子材料 COPs, 但由于缺乏刚性骨架 以及立体支撑结构, 基于间苯三酚制备的高分子 COP-
36 的 BET 比表面积仅为 $11 \mathrm{~m}^{2} \cdot \mathrm{g}^{-1}$ (表 1, Entry 3). 2017 年, Kejik 等 ${ }^{[66]}$ 以醋酸硅与间苯三酚高温缩合制备了有 机多孔芳香族硅酸盐凝胶, 醋酸硅的立体结构起到了关 键的骨架支撑作用, 其最大 BET 表面积可达 $990 \mathrm{~m}^{2}$ • $\mathrm{g}^{-1}$, 并具有良好的热稳定性, 在催化、气体分离与储存 等领域中有着可观的应用前景(表 1, Entries 4 6). 2018 年, 谭必恩课题组 ${ }^{[67]}$ 以间苯三酚为核心, 利用 Mannich 反应与芳香二胺、甲醛反应制备了苯并噁嗪环连接的杂 核多孔高分子材料 BoxPOP-1, BoxPOP-2 和 BoxPOP3(表 1, Entry 7 9), 其中 BoxPOP-1 的 BET 比表面积可 达 $231 \mathrm{~m}^{2} \cdot \mathrm{g}^{-1}$, 其最大 $\mathrm{CO}_{2}$ 吸附量可达 $1.55 \mathrm{mmol} \cdot \mathrm{g}^{-1}$ $(273 \mathrm{~K}, 100 \mathrm{kPa})$. 马利建课题组 ${ }^{[68]}$ 以间苯三酚与六氯环 三磷腈缩聚得到高性能铀吸附材料 CPF-T(表 1, Entry 
10), 其 BET 表面积可达 $654 \mathrm{~m}^{2} \cdot \mathrm{g}^{-1}$ 并展示出 $0.20 \sim 0.26$ $\mathrm{mmol} \cdot \mathrm{g}^{-1}$ 的高吸附量 $(\mathrm{pH}=4$ 的弱酸性环境到 $3 \mathrm{~mol} / \mathrm{L}$ $\mathrm{HNO}_{3}$ 的强酸性环境), 并在 $3 \mathrm{~mol} / \mathrm{L} \mathrm{HNO}_{3}$ 的强酸性环境 中表现出 $72 \%$ 的铀吸附选择性. 该工作为固相萃取剂 (SPE)的研究制备提供了新的思路.

基于间苯三酚羟基的 $\mathrm{O}$-功能化反应策略，人们发 展了诸多性能优良的有机多孔高分子材料, 具有 $\mathrm{CO}_{2}$ 吸 附及气体分离等方面的应用潜力. 但该策略也有不足之 处, 首先, 相比普通有机多孔高分子材料, 其聚合过程 往往需要高温或长时间反应(王忠刚课题组 ${ }^{[62]}$ 报道的聚 合反应需要 $170{ }^{\circ} \mathrm{C}$ 高温下反应 $4 \mathrm{~h}$, Ullah 等 ${ }^{[65]}$ 报道的聚 合反应需要 $24 \mathrm{~h}$ 的反应时间); 其次, 以该策略构筑的 多孔高分子由于单体尺寸限制等原因往往缺乏规则的 孔道结构, 因此比表面积与孔性能不突出, 对材料性能 及应用存在一定影响. 优化反应条件、增大单体尺寸或 是引入立体结构是该策略需要提升改进的地方.

\section{2 基于间苯三酚中苯环的 C-功能化反应策略构筑多 孔高分子}

由于多羟基的供电子作用，间苯三酚也能通过富电 子苯环的 C-功能化来制备保留羟基(或着基异构形式)的 不同键接方式的多孔高分子材料, 从而大大拓展了合成
设计思路. 目前，间苯三酚的直接 C-功能化反应策略主 要包括醛基缩合与偶氮偶联聚合反应等.

醛基缩合反应类似于酚醛树脂的合成，利用多元醛 进攻间苯三酚苯环 $\mathrm{H}$ 原子，脱水形成 $\mathrm{C}-\mathrm{C}$ 键构筑多孔 高分子材料(Scheme 10). 2011 年, Katsoulidis 等 ${ }^{[69]}$ 以对 苯二甲醛、4-(4-甲酰基苯氧基)苯甲醛以及三(4-甲酰基 苯基)胺与间苯三酚缩聚制备了一系列新型有机聚合框 架 $($ POF1A $\sim$ POF3A, POF1B $\sim$ POF3B, 表 2, Entries 1 $3)$, 其中对苯二甲醛与间苯三酚经缩合、高温活化得到 的 POF1B 具有高达 $917 \mathrm{~m}^{2} \cdot \mathrm{g}^{-1}$ 的 BET 比表面积, 平均 孔径仅为 $0.6 \mathrm{~nm}, \mathrm{C}-\mathrm{C}$ 键接的聚合骨架使其在较宽的 $\mathrm{pH}$ 范围 $(\mathrm{pH}=1 \sim 13)$ 内呈现可观的化学稳定性, 而多孔 的多羟基芳环网络体系还提供了气体吸附应用(在 273 $\mathrm{K}, 100 \mathrm{kPa}$ 条件下 $\mathrm{CO}_{2}$ 吸附量达 $4.09 \mathrm{mmol} \cdot \mathrm{g}^{-1}$ ) 以及潜 在的半导体应用性能(光学吸收带隙为 $1.5 \sim 2.5 \mathrm{eV}$ ), 这 类醛基缩合反应制备的有机多孔高分子材料展现出了 巨大的应用潜力. 次年，在此前工作基础上 Katsoulidis 等 ${ }^{[70]}$ 在 POF1B 合成过程中引入溴化十六烷基吡啶鎓链 段，制备得到了 meso- POF 材料，且不同反应条件得到 $1.5 \sim 2.5 \mathrm{~nm} 、 2 \sim 5 \mathrm{~nm} 、 2 \sim 2.5 \mathrm{~nm}$ 与 $10 \sim 25 \mathrm{~nm}$ 三种不 同孔径分布, 最高 BET 比表面积达到 $1027 \mathrm{~m}^{2} \cdot \mathrm{g}^{-1}$ (表 2,

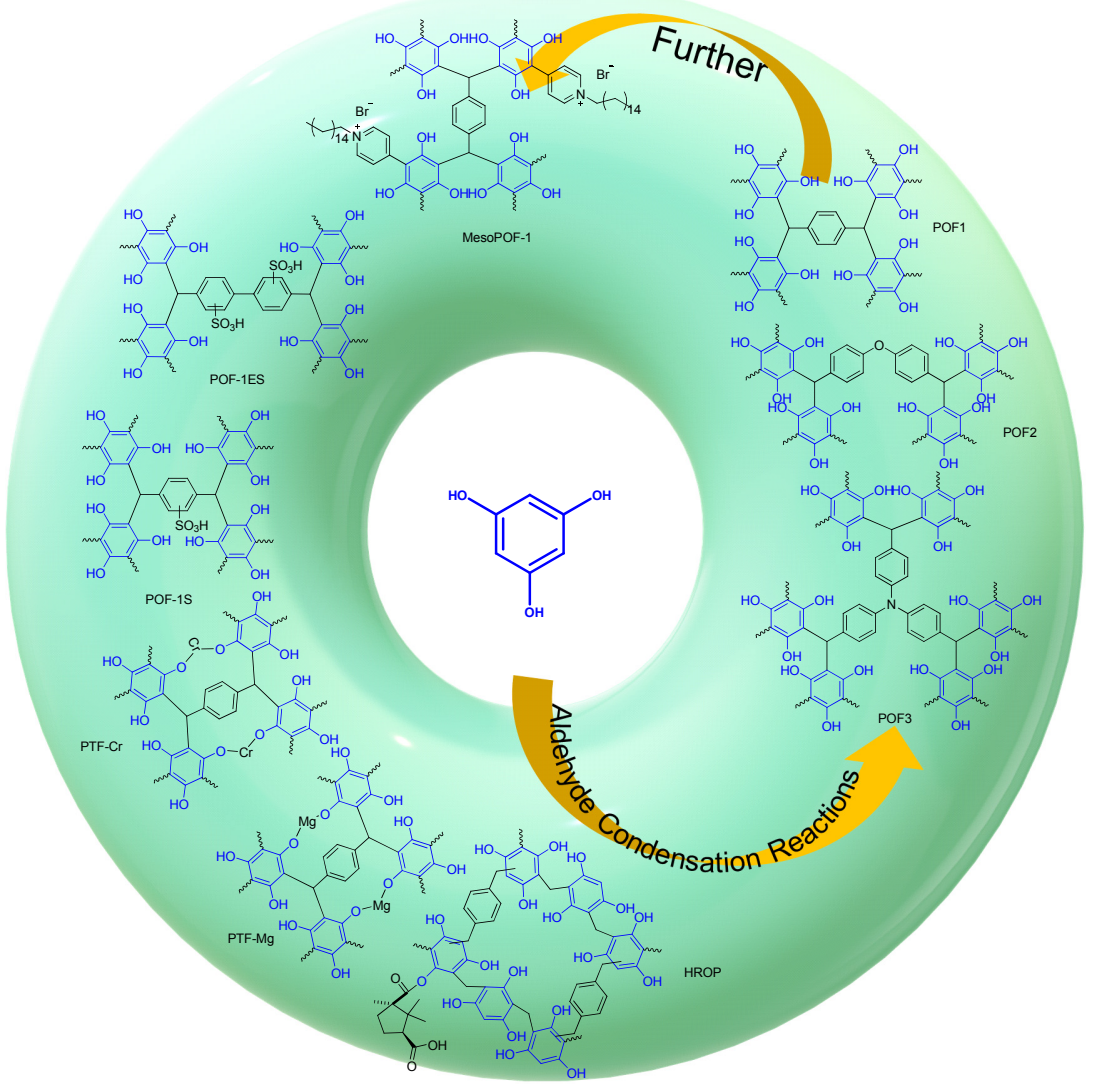

图式 10 基于醛基缩合反应的 POPs

Scheme 10 POPs based on aldehyde condensation reactions 
表 2 基于醛基缩合反应的 POPs 总结

Table 2 Summary of POPs based on aldehyde condensation reactions

\begin{tabular}{lllcc}
\hline Entry & Polymer & $S_{\mathrm{BET}} /\left(\mathrm{m}^{2} \cdot \mathrm{g}^{-1}\right)$ & Mean pore size $/ \mathrm{nm}$ & $V_{\mathrm{tot}} /\left(\mathrm{g} \cdot \mathrm{cm}^{-3}\right)$ \\
\hline $1^{[69]}$ & POF1B, POF1A & 917,773 & 0.6 & - \\
$2^{[69]}$ & POF2B, POF2A & 769,658 & 0.6 & - \\
$3^{[69]}$ & POF3B, POF3A & 608,115 & - & 0.6 \\
$4^{[70]}$ & MesoPOF-1 & 1027 & - & - \\
$5^{[71]}$ & PRP-1 & 835 & - & - \\
$6^{[73]}$ & POF-1S & 29 & - & - \\
$7^{[72]}$ & PTF-Cr & 106 & - & - \\
$8^{[72]}$ & PTF-Mg & 128 & - & - \\
$9^{[74]}$ & POF-1ES & 57 & - & 0.80 \\
$10^{[76]}$ & HROP & 568 & - \\
\hline
\end{tabular}

Entry 4), 并且对 $\mathrm{C}_{2} \mathrm{H}_{6}$ 和 $\mathrm{CH}_{4}$ 表现出极高的选择性吸附 性能, 在 $273 \mathrm{~K}, 100 \mathrm{kPa}$ 条件下 $\mathrm{C}_{2} \mathrm{H}_{6} / \mathrm{CH}_{4}$ 选择比可达 40[依据 IAST (ideal adsorbed solution theory)模型计算]. 此后, 醛基缩合制备基于间苯三酚的多孔材料吸引了更 多研究者, 材料的合成与应用性能得到更进一步的研 究.

2016 年, 江海龙课题组 ${ }^{[71]}$ 以 “一锅法” 实现 4,4'联苯二甲醛与间苯三酚的缩聚, 得到了具有优良稳定性 和高比表面积 $\left(S_{\mathrm{BET}}=835 \mathrm{~m}^{2} \cdot \mathrm{g}^{-1}\right)$ 的多孔高分子材料 PRP-1, 其以微孔为主(小于 $2 \mathrm{~nm}$ ), 兼有介孔、大孔的孔 径分布特性, 有利于实现 $\mathrm{CO}_{2}$ 的高效吸附 $(273 \mathrm{~K}, 100$ $\mathrm{kPa}$ 条件下吸附量达 $2.09 \mathrm{mmol} \cdot \mathrm{g}^{-1}$ )与催化转化(表 2 , Entry 5). Pareek 等 ${ }^{[72]}$ 向对苯二甲醛与间苯三酚的高分子 中引入 $\mathrm{Cr}$ 和 $\mathrm{Mg}$, 形成了多孔高分子配合物 PTF-Cr 和 $\operatorname{PTF}-\mathrm{Mg}\left(\right.$ 表 2, Entries 7,8), 直接暴露在外的金属与 $\mathrm{H}_{2}$ 较强的相互作用赋予了高分子优秀的储氢性能，在 298 $\mathrm{K}$ 和 $10.1 \mathrm{MPa}$ 条件下二者分别能储存质量分数为 $1.5 \%$ 和 $0.5 \%$ 的 $\mathrm{H}_{2}$. Kang 等 ${ }^{[73-75]}$ 分别以对苯二甲醛和 4,4'-联 苯二甲醛同间苯三酚缩聚, 再进行苯环磺酸化修饰, 制 备了具有高电导率的有机多孔高分子材料(在 $80{ }^{\circ} \mathrm{C}$ 和 $90 \%$ 相对湿度条件下，电导率分别为 $7.72 \times 10^{-2}$ 和 1.59 $\left.\times 10^{-1} \mathrm{~S} \cdot \mathrm{cm}^{-1}\right)$, 并进一步利用该磺酸化修饰的酚醛高 分子与聚偏二氟乙烯(PVDF)复合制膜, 获得了高电导 率的膜材料 (在 $70^{\circ} \mathrm{C}$ 和 $90 \%$ 相对湿度条件下, 电导率达 到 $2.13 \times 10^{-2} \mathrm{~S} \cdot \mathrm{cm}^{-1}\left(70{ }^{\circ} \mathrm{C}\right.$ 和 $90 \%$ 相对湿度条件下), 进一步拓展了导电多孔高分子的设计思路(表 2, Entries $6,9)$.

刘洪来等 ${ }^{[76]}$ 则利用甲醛与间苯三酚缩聚构筑了类 酚醛树脂结构, 并利用三嵌段共聚物 F127(聚氧乙烯-聚 氧丙烯-聚氧乙烯共聚物)与间苯三酚形成氢键, 诱导形 成微孔结构, 再以对二氯甲基苯进行超交联处理, 得到 多孔高分子 $\operatorname{HROP}($ 表 2, Entry 10), 其经 $D$-樟脑酸酯化 手性修饰后与硅胶片复合得到的 HROP@SGS 材料, 能 够在 $30 \mathrm{~min}$ 内完成对 1-苯乙醇、布洛芬、荎普生手性
成分的有效分离, 其分离度 $\left(R_{\mathrm{s}}\right)$ 均大于 $1.5,1$-苯乙醇的 分离因子 $(\alpha)$ 可达 11.54 , 具有手性分子分离的实际应用 潜力. 醛基缩合反应能够得到(超)微孔结构的交联多孔 高分子, 其在吸附、分离等领域展示出了优秀的应用潜 力与性能，基于该反应策略制备更多高性能多孔高分子 有待更深入的研究.

重氮偶联反应是多元胺制备的重氮盐与间苯三酚 发生的亲电取代反应，能够形成偶氮键接的多孔高分子 (Scheme 11). 相比醛基缩合反应以及制备同样偶氮类高 分子的硝基还原偶联反应，其反应更温和，更易实现， 同时引入的偶氮键赋予材料更高的功能性, 因此以重氮 偶联反应构筑偶氮类有机多孔高分子材料近年吸引到 了越来越多的关注.

2016 年, 刘志敏课题组 ${ }^{[77]}$ 尝试以多元芳香胺制备 的重氮盐与多元芳香酚发生重氮偶联反应，制备了偶氮 类有机多孔高分子，并表征了其多孔特性，其中间苯三 酚与 4,4'-二氨基联苯、1,3,5-三(4-氨苯基)苯制备得到的 HAzo-POP-1 和 HAzo-POP-3(表 3, Entries 1, 2)均以介孔 为主并伴有少量微孔分布, 在多氮基团与孔结构的双重 作用下该偶氮类有机多孔高分子对 $\mathrm{CO}_{2}$ 的吸附与催化 环加成转化表现出可观的潜力. 胡斌等 ${ }^{[78]}$ 以 $4,4^{\prime}$-二氨基 联苯重氮盐与间苯三酚重氮偶联后负载 $\mathrm{Fe}_{3} \mathrm{O}_{4} @ \mathrm{SiO}_{2}$ 纳 米颗粒得到了介孔结构的磁性有机多孔高分子材料 MOP-2, 由于甲基蓝与间苯三酚结构的氢键作用, 该材 料对甲基蓝染料的吸附量达 $1153 \mathrm{mg} \cdot \mathrm{g}^{-1}$, 并易于回收 重复利用(5 次循环使用吸附量未见明显衰减). 2018 年, Bhat 等 ${ }^{[79]}$ 以菱二酰亚胺二胺重氮盐与间苯三酚重氮偶 联后负载 $\mathrm{Co}^{2+}$ 得到的有机多孔高分子材料 $\mathrm{Co}^{\mathrm{II}}-\mathrm{NDI}-\mathrm{PG}$ 作为析氧反应(OER)电催化剂(表 3, Entry 3). 得益于共 轭网络结构与负载金属的共同作用, 在碱性环境中 $(\mathrm{pH}=13.6)$, 过电位达到 $340 \mathrm{mV}$ 时即可获得 $1 \mathrm{~mA} \cdot \mathrm{cm}^{-2}$ 的催化电流密度, 且 Tafel 斜率仅为 $68 \mathrm{mV} / \mathrm{decade}$. 许彦 红等 ${ }^{[80]}$ 以四(4-氨基苯基)乙烯重氮盐与间苯三酚重氮偶 联制得了共轭微孔高分子 AzoTPE-CMP(表 3, Entry 4), 


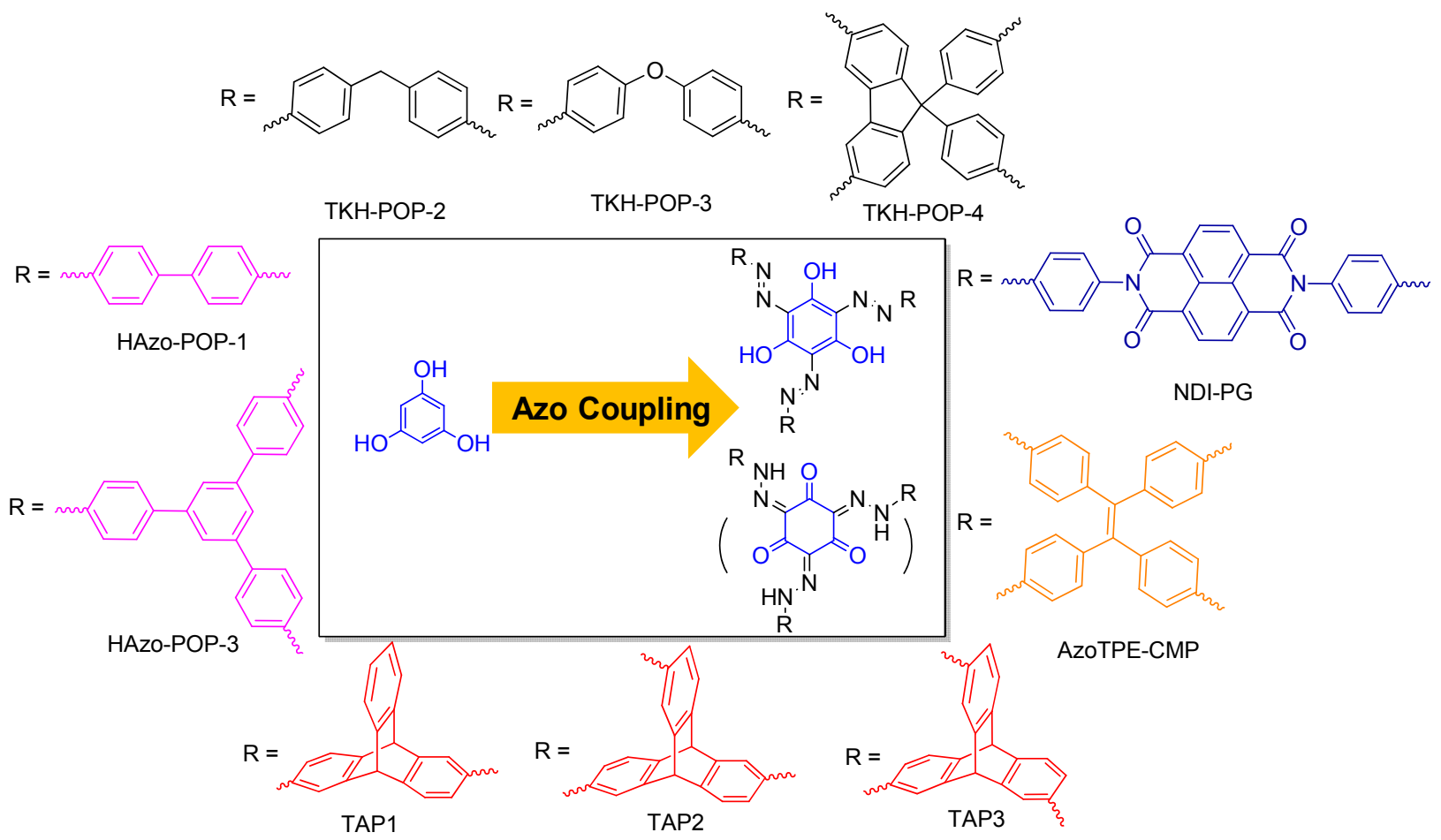

图式 11 基于重氮偶联反应的 POPs

Scheme 11 POPs based on azo coupling reactions

表 3 基于重氮偶联反应的 POPs 总结

Table 3 Summary of POPs based on azo coupling reactions

\begin{tabular}{lllll}
\hline Entry & Polymer & $S_{\mathrm{BET}} /\left(\mathrm{m}^{2} \cdot \mathrm{g}^{-1}\right)$ & Mean pore size $/ \mathrm{nm}$ & $V_{\text {tot }} /\left(\mathrm{g} \cdot \mathrm{cm}^{-3}\right)$ \\
\hline $1^{[77]}$ & HAzo-POP-1 & 256 & - & 1.325 \\
$2^{[77]}$ & HAzo-POP-3 & 418 & - & 1.730 \\
$3^{[79]}$ & Co ${ }^{\text {II }}$ NDI-PG, NDI-PG & 88,93 & - & - \\
$4^{[80]}$ & Ag@AzoTPE-CMP, AzoTPE-CMP & 47,366 & - & $0.110,1.072$ \\
$5^{[81]}$ & TAP1 & 474 & - & 0.74 \\
$6^{[81]}$ & TAP2 & 772 & - & 1.41 \\
$7^{[81]}$ & TAP3 & 729 & - & 1.04 \\
$8^{[22]}$ & TKH-POP-2 & 437 & - & 1.4 \\
$9^{[22]}$ & TKH-POP-3 & 552 & - & 2.3 \\
$10^{[22]}$ & TKH-POP-4 & 639 & 2.2 \\
\hline
\end{tabular}

其 BET 比表面积为 $366 \mathrm{~m}^{2} \cdot \mathrm{g}^{-1}$, 负载 $\mathrm{Ag}^{+}$后下降到 47 $\mathrm{m}^{2} \cdot \mathrm{g}^{-1}$, 但表现出对碘蒸汽较高的吸附能力 (在 $350 \mathrm{~K}$ 条 件下 AzoTPE-CMP 与 Ag@AzoTPE-CMP 对碘蒸汽的吸 附量分别为 1991 和 $2598 \mathrm{mg} \bullet \mathrm{g}^{-1}$ ) 和对重金属离子如 $\mathrm{Cu}^{2+}, \mathrm{Hg}^{2+}, \mathrm{Cr}^{3+}, \mathrm{Ni}^{2+}$ 等的探测能力. 2019 年, Bera 等 ${ }^{[81]}$ 以具有三维结构的三蝶烯重氮盐与间苯三酚重氮偶联 得到了有机多孔高分子材料 TAPs(表 3, Entries 5～7), 在多氮基团与孔结构的共同作用下表现出对较高的 $\mathrm{CO}_{2}$ 吸附能力与选择性(在 $273 \mathrm{~K}, 100 \mathrm{kPa}$ 条件下吸附量 最高达 $3.41 \mathrm{mmol} \cdot \mathrm{g}^{-1}, \mathrm{CO}_{2} / \mathrm{CH}_{4}$ 选择比为 114$)$.

黄木华课题组 ${ }^{[22]}$ 通过二维核磁证明了间苯三酚的 偶氮化产物存在三 $(\beta$-羟基-偶氮 $)$ 苯到三 $(\beta$-酮 - 氢偶氮 $)$ 环己烷结构的互变异构现象, 并以此构筑了基于间苯三
酚的偶氮类有机多孔高分子材料 TKH-POPs(表 4, Entries $8 \sim 10$ ), 其中 TKH-POP-4 的 BET 比表面积最大可 达 $639 \mathrm{~m}^{2} \cdot \mathrm{g}^{-1}$, 而 TKH-POP-1 在 $298 \mathrm{~K}$ 和 $p / p_{0}=0.98$ 条 件下对乙腈蒸汽的吸附量高达 $66.3 \mathrm{mmol} \cdot \mathrm{g}^{-1}$. 这些研 究充分展现了重氮偶联反应构筑的基于间苯三酚偶氮 类有机多孔高分子材料的应用潜力.

除了上述介绍的两类反应外, 研究者们还通过如 Friedel-Craft 反应等其他的亲电反应进行 C-功能化以构 筑不同结构的多孔高分子. 2015 年, Vinodh 等 $^{[82]}$ 利用 Friedel-Craft 反应以间苯三酚与不同物质的量的对二氯 甲基苯聚合得到超交联高分子 HCP-1 HCP-4 (Scheme $12)$, 其中 HCP-4 具有最大 BET 比表面积 $\left(634 \mathrm{~m}^{2} \cdot \mathrm{g}^{-1}\right)$, 为利用低成本单体构筑高性能有机多孔高分子材料提 
供了参考. 2016 年, 张肜课题组 ${ }^{[83]}$ 利用 Friedel-Craft 反 应以间苯三酚与二甲氧基甲烷聚合得到了分级多孔高 分子微球(Scheme 12), 其中交联网络产生了 $0.65 \mathrm{~nm}$ 的 微孔结构, 高分子纳米颗粒堆积产生了 $2.57 \mathrm{~nm}$ 的介孔 结构, 微孔与介孔结构均能够实现水分子的高效传输, 同时介孔结构还能实现 $\mathrm{LiCl}$ 的均匀负载, 该高分子微 球仅需负载质量分数为 $3 \%$ 的 $\mathrm{LiCl}$ 即能实现在 $11 \%$ 95\%的相对湿度环境下高灵敏度的湿度探测.

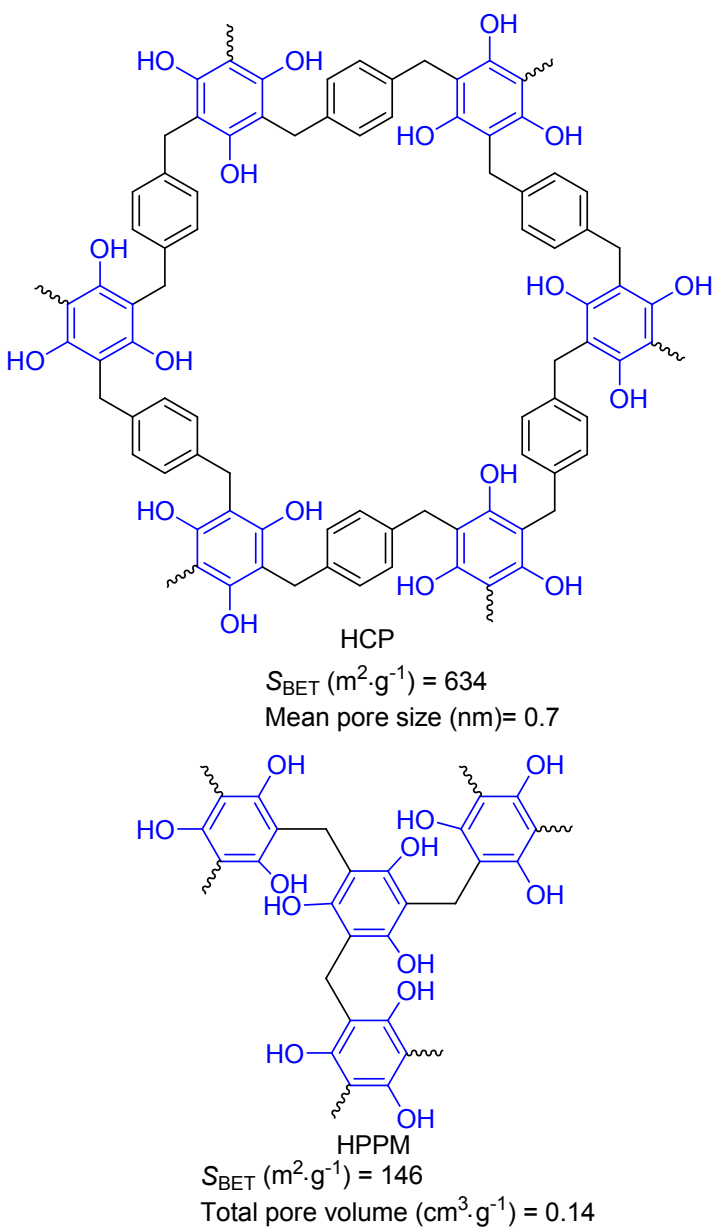

图式 12 基于 Friedel-Craft 反应的 POPs

Scheme 12 POPs based on Friedel-Craft reactions

如今苯环 C-功能化反应策略主要建立在重氮偶联 与醛基取代反应基础上, 能够得到具有均匀孔道结构与 理想比表面积的非晶态多孔高分子(BET 比表面积往往 在 $300 \sim 1000 \mathrm{~m}^{2} \cdot \mathrm{g}^{-1}$ 之间), 并且原料成本低廉, 反应相 对温和快速(尤其是可常温反应的重氮偶联反应), 所构 筑的高分子在气体吸附等领域应用表现突出. 同时 Friedel-Craft 反应等其他类型的亲电反应也被应用到高 性能新型多孔高分子的合成中, 为高效构筑新型有机多 孔高分子提供了更多合成思路与可能. 苯环 C-功能化 反应策略在基于间苯三酚及其衍生物的新型多孔高分 子合成设计应用中展示出优秀的适用性, 仍有大量高性
能多孔高分子材料等待进一步研究发掘.

\section{3 基于间苯三酚衍生物的聚合反应策略构筑多孔高 分子}

除了以间苯三酚的直接功能化反应制备多孔高分 子外，基于间苯三酚衍生物的聚合反应进一步拓宽了功 能性多孔高分子的设计思路(Scheme 13). 2013 年, 朱广 山课题组 ${ }^{[84]}$ 以间苯三酚的三溴代衍生物三溴间苯三酚 (12)与四(4-乙炔基苯基)甲烷聚合得到三维网状结构的 PAF-18 系列多孔高分子(表 4, Entry 1), 与金属 Li 结合 后能实现对 $\mathrm{CO}_{2}$ 和 $\mathrm{H}_{2}$ 的高效吸附(其中在 $273 \mathrm{~K}, 100$ $\mathrm{kPa}$ 条件下 $\mathrm{CO}_{2}$ 吸附量可达 $\left.3.27 \mathrm{mmol} \cdot \mathrm{g}^{-1}\right)$. 他们 ${ }^{[85]}$ 还 尝试了三溴间苯三酚与三(4-乙炔基苯)胺的聚合，得到 了 PAF- 34-OH 多孔高分子(表 4, Entry 2), 羟基的存在 增大了材料与 $\mathrm{CO}_{2}$ 的相互作用, 从而同样表现出较好的 $\mathrm{CO}_{2}$ 的吸附性能(其中在 $273 \mathrm{~K}, 100 \mathrm{kPa}$ 条件下 $\mathrm{CO}_{2}$ 吸附 量可达 $\left.2.21 \mathrm{mmol} \cdot \mathrm{g}^{-1}\right)$. 随后, 该课题组 ${ }^{[86]}$ 利用三澳间 苯三酚与三乙炔基苯设计合成了 PAF-80 和对羟基进行 磺酸化修饰的 PAF-80- $\mathrm{SO}_{3} \mathrm{H}$ 多孔高分子(表 4, Entry 3), 丰富的羟基、磺酸基团赋予了高分子增强的极性，使 PAF-80 和 PAF-80-SO $\mathrm{S}_{3} \mathrm{H}$ 分别具有 194 和 $226 \mathrm{~cm}^{3} \cdot \mathrm{g}^{-1}$ 的 吸水性能, 并为高分子极性控制与水体污染物吸附提供 了思路. 2017 年, Patra 等 ${ }^{[87]}$ 利用 Duff 反应向间苯三酚中 引入醛基得到 TFP, 再与 5,10,15,20-四(4-氨基苯基)$21 H, 23 H$-卟啉发生席夫碱生成反应，聚合得到的非晶态 的 COP 材料作为析氢反应的非金属催化剂(表 4, Entry 4), 在 $250 \mathrm{mV}$ 的过电位下即获得 $10 \mathrm{~mA} \cdot \mathrm{cm}^{-2}$ 的还原电 流密度, 且 Tafel 斜率仅为 $106 \mathrm{mV} / \mathrm{decade}$. 与之类似, Bhanja 等 ${ }^{[88}$ 采用 Vilsmeier-Haack 反应向间苯三酚引入 醛基得到二甲醛间苯三酚(13), 其与 1,3,5-三(4-氨基苯 基)三嗪缩聚得到的多孔高分子 TPDA-1(表 4, Entry 5), 在 $2 \mathrm{mV}$ 的扫描速度下展示出 $469.4 \mathrm{~F} \cdot \mathrm{g}^{-1}$ 的高比电容值. 类似的, Das 等 ${ }^{\left[{ }^{89]}\right.}$ 以间苯三酚合成的二甲醛间苯三酚与 1,4-二(4,6-二氨基- $s$-三嗪-2-基)-苯缩聚得到的多孔高分 子 TrzPOP-3(表 4, Entry 6), 在 $273 \mathrm{~K}, 100 \mathrm{kPa}$ 条件下 $\mathrm{CO}_{2}$ 吸附量高达 $8.54 \mathrm{mmol} \cdot \mathrm{g}^{-1}$, 其 $\mathrm{CO}_{2} / \mathrm{N}_{2}$ 吸附选择比 可达 167.4(依据 IAST 模型计算), 并通过理论计算发现 含有丰富亲电基团(如酚羟基、 $\mathrm{N}$ 原子)和高比表面积是 提高 $\mathrm{CO}_{2}$ 吸附量的关键, 为后续 $\mathrm{CO}_{2}$ 吸附新材料的开发 提供了设计参考思路. Kochergin 等 ${ }^{[90-91]}$ 以由间苯三酚合 成的苯并三噻吩类衍生物 (BTT, 14) 作为电子给体, 2,4,6-三氯-1,3,5-三嗪(1)、4,6-三(4-溴苯基)-1,3,5-三嗪(2) 作为电子受体, 通过给体一受体相互作用成键聚合, 得 到了硫氮杂原子掺杂的有机多孔高分子 SNP-BTT1 和 SNP-BTT2(表 4, Entries 7,8), 其共轭网络结构赋予了高 分子突出的电荷传导能力, 在光催化和传感器等多个领 


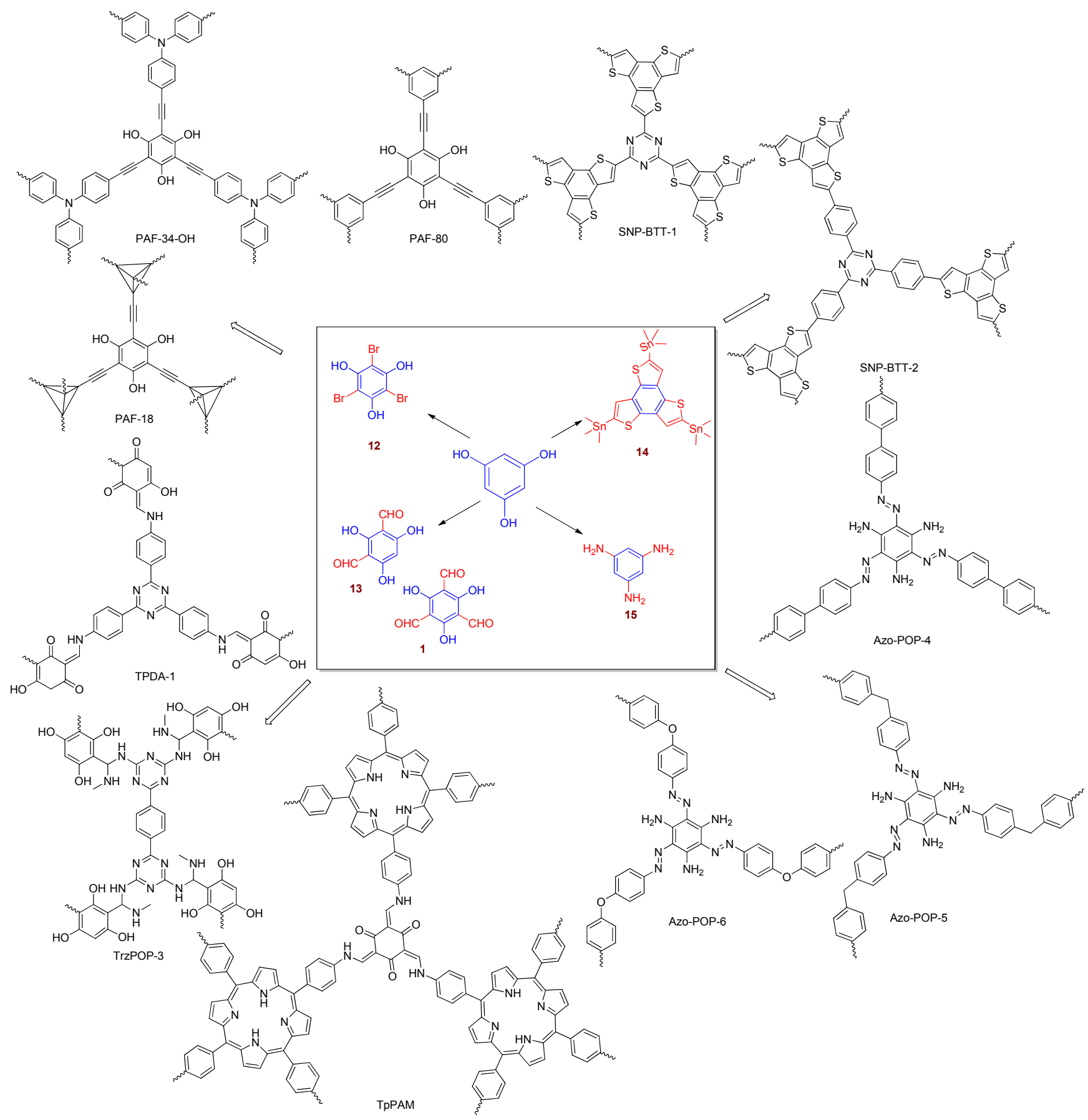

图式 13 基于间苯三酚衍生物聚合反应的 POPs

Scheme 13 POPs based on polymerization of derivatives of phloroglucinol

域具有应用研究潜力. 2019 年, 黄木华课题组 ${ }^{[1]}$ 从间苯 三酚出发逆向合成得到了 1,3,5-三氨基苯(15), 并使其 与芳香二胺(苯胺、对甲基苯胺、对甲氧基苯胺)重氮盐 进行重氮偶联，制备了有机多孔高分子 Azo-POP-4, Azo-POP-5 和 Azo-POP-6(表 4, Entries 9 11), 其介孔结 构有利于金属负载以及小分子的快速传输, 负载 $\mathrm{Cu}^{2+}$ 后能实现高效的 $\mathrm{C}-\mathrm{H}$ 键催化氧化反应. 基于间苯三酚 衍生物的聚合反应策略不拘泥于某几种化学反应过程, 能有效丰富高性能单体库和聚合反应种类, 从而制备出
具有各种特殊结构的高分子, 并带来优秀的应用性能 (如制备全共轭网络高分子实现电化学相关应用). 但这 一策略主要依靠合成经验，单体设计合成与高分子设计 合成相比其他策略往往需要更多的精力投入.

而基于间苯三酚及其衍生物的 O-功能化反应、C功能化反应以及衍生物聚合反应策略, 已经成功构筑了 一系列高性能有机非晶态多孔高分子材料，它们在能 源、催化、气体吸附与分离等领域均展示出可观的应用 价值. 其中, 间苯三酚的 O-功能化反应能够构筑多种成 
表 4 基于间苯三酚衍生物聚合反应 POPs 总结

Table 4 Summary of POPs based on polymerization of derivatives of phloroglucinol

\begin{tabular}{lllll}
\hline Entry & Polymer & $\begin{array}{l}S_{\mathrm{BET}} / \\
\left(\mathrm{m}^{2} \bullet \mathrm{g}^{-1}\right)\end{array}$ & $\begin{array}{l}\text { Mean pore } \\
\text { size/nm }\end{array}$ & $\begin{array}{l}V_{\text {tot }} \\
\left(\mathrm{g} \bullet \mathrm{cm}^{-3}\right)\end{array}$ \\
\hline $1^{[84]}$ & PAF-18 & 1121 & 1.33 & 0.82 \\
& PAF-18-OLi & 982 & 1.32 & 0.70 \\
$2^{[85]}$ & PAF-34-OH & 771 & - & - \\
$3^{[86]}$ & PAF-80 & 768 & - & - \\
$4^{[87]}$ & TpPAM & 654 & 1.6 & 0.23 \\
$5^{[88]}$ & TPDA-1 & 545 & - & 0.255 \\
$6^{[89]}$ & TrzPOP-3 & 772 & - & - \\
$7^{[90]}$ & SNP-BTT-1 & 698 & - & - \\
$8^{[90]}$ & SNP-BTT-2 & 411 & - & - \\
$9^{[61]}$ & Azo-POP-4 & 368 & 25.7 & 1.809 \\
$10^{[61]}$ & Azo-POP-5 & 196 & 38.2 & 1.784 \\
$11^{[61]}$ & Azo-POP-6 & 210 & 35.0 & 1.776 \\
\hline
\end{tabular}

键类型(如硅醚、磷醚等 ${ }^{[66,68]}$ ) 的有机多孔材料, 其合成 途径易于设计. 其 C-功能化反应能够构筑保留羟基基 团的多孔骨架材料, 使得间苯三酚多羟基优势得以充分 体现，在吸附等领域展现出极为优异的性能和应用前 景. 其衍生物聚合反应则大大丰富了多官能度有机单体 选择和高分子构筑设计思路, 能够选择性保留羟基并引 入新官能团实现功能化, 从而使高分子性能大为拓展, 如具有优秀的半导体性能 ${ }^{[90]}$.

未来将间苯三酚的 $\mathrm{O}$-功能化反应、C-功能化反应以 及衍生物聚合反应策略综合应用, 从化学合成角度引导 材料设计, 或能推动基于间苯三酚的非晶态有机多孔材 料合成设计与应用更进一步的研究.

\section{3 总结与展望}

综上所述，基于间苯三酚及其衍生物的不同反应策 略，人们已经发展了一系列结构新颖的有机多孔材料, 例如基于间苯三酚衍生物 TFP 构筑的烯胺类 COFs 材

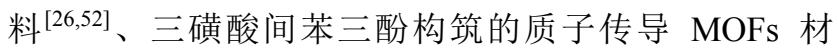

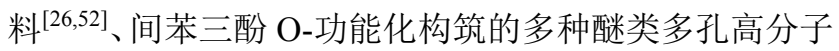
材料 ${ }^{[66,68]}$ 以及 $\mathrm{C}$-功能化构筑的酚醛树脂类 POF 材料和 偶氮类多孔高分子材料 ${ }^{[69,77]}$ 等. 利用间苯三酚及其衍生 物的优势反应平台, 能够构筑 $-\mathrm{C}-\mathrm{O}-\mathrm{C} 、-\mathrm{C}-\mathrm{O}-$ $\mathrm{P}-、-\mathrm{C}-\mathrm{C}-、-\mathrm{C}=\mathrm{N}-、-\mathrm{C}-\mathrm{N}-、-\mathrm{N}=\mathrm{N}-$ 等 多种不同化学键接的有机多孔材料, 这些不同的成键方 式对于有机多孔材料最终的性能有着重要影响. 马利建 课题组 ${ }^{[68]}$ 以间苯三酚与六氯环三磷腈构筑了- $\mathrm{C}-\mathrm{O}-$ $\mathrm{P}$ 一键接的多孔高分子材料, 在高键能的 $\mathrm{P}-\mathrm{O}$ 键与 $\mathrm{d}-\mathrm{p}$ 反馈 $\pi$ 键的协同作用下, 该材料在强酸性环境下仍能实 现高效稳定的铀吸附应用. Baek 等 $^{[42]}$ 以 HAB 与环己六 酮构筑的含 $\mathrm{C}=\mathrm{N}$ 键接结构的氮化碳材料 $\mathrm{C}_{2} \mathrm{~N}$ 中, 杂原 子的引入使材料产生了带隙, 从而具备了 $\mathrm{sp}^{2}$ 碳构筑的
石墨烯所没有的半导体性能. 此外，间苯三酚自身的酚 差弪基除了参与材料骨架的构筑，对材料性能也有着重要 影响, Banerjee 等 ${ }^{[26]}$ 以 TFP 构筑了 $\mathrm{TpPa}$ 系列 COFs 材料, 酚差基的存在得以使 COFs 不稳定的亚胺结构转化为稳 定的烯胺结构, 大幅提升了 COFs 材料的化学稳定性. 类似的, Baek 等 ${ }^{[41]}$ 以 TAPG 构筑的亚胺 COFs 材料通过 与邻位羟基的进一步反应实现不可逆噁唑环的构筑，同 样大幅提升了 COFs 材料的化学稳定性. 酚羟基的存在 对于材料吸附性能同样有着重要贡献 ${ }^{[69,78,85]}$. 因此，间 苯三酚及其衍生物作为优秀的平台分子在新型高性能 有机多孔材料构筑领域有着巨大的应用潜力, 利用间苯 三酚 $\mathrm{O}$-功能化反应、C-功能化反应、衍生物聚合反应等 策略进一步挖掘已有的基于间苯三酚及其衍生物的有 机多孔材料的应用价值, 以及巧妙借助有机合成化学中 的人名反应，进一步发展间苯三酚衍生的优秀平台分子 和高效的聚合反应，创造结构新颖的有机多孔材料，并 拓展其应用领域, 将成为未来一段时间的研究热点.

\section{References}

[1] Sanna, C.; Scognamiglio, M.; Fiorentino, A.; Corona, A.; Graziani, V.; Caredda, A.; Cortis, P.; Montisci, M.; Ceresola, E. R.; Canducci, F.; Poli, F.; Tramontano, E.; Esposito, F. PLoS One 2018, 13, e195168.

[2] Broadley, K.; Larsen, L.; Herst, P. M.; Smith, R. A. J.; Berridge, M. V.; Mcconnell, M. J. J. Cell Biochem. 2011, 112, 1869.

[3] Zhang, B. X.; Duan, D. Z.; Ge, C. P.; Yao, J. P.; Liu, Y. P.; Li, X. M.; Fang, J. G. J. Med. Chem. 2015, 58, 1795.

[4] Bellamy, A. J.; Ward, S. J.; Golding, P. Propellants, Explos., Pyrotech. 2002, 27, 49 .

[5] Wurzenberger, M. H. H.; Bissinger, B. R. G.; Lommel, M.; Gruhne, M. S.; Szimhardt, N.; Stierstorfer, J. New J. Chem. 2019, 43, 18193.

[6] Liang, C. D.; Dai, S. J. Am. Chem. Soc. 2006, 128, 5316.

[7] Yuan, F. L.; Yuan, T. S.; Sui, L. Z.; Wang, Z. B.; Xi, Z. F.; Li, Y. C.; Li, X. H.; Fan, L. Z.; Tan, Z. A.; Chen, A. M.; Jin, M. X.; Yang, S. H. Nat. Commun. 2018, 9, 2249.

[8] Tsyurupa, M. P.; Davankov, V. A. React. Funct. Polym. 2002, 53, 193.

[9] Wang, S.; Song, K.; Zhang, C.; Shu, Y.; Li, T.; Tan, B. J. Mater Chem. A 2017, 5, 1509.

[10] Tan, L. X.; Tan, B. E. Chem. Soc. Rev. 2017, 46, 3322.

[11] Chen, L.; Honsho, Y.; Seki, S.; Jiang, D. L. J. Am. Chem. Soc. 2010, 132, 6742 .

[12] Dawson, R.; Adams, D. J.; Cooper, A. I. Chem. Sci. 2011, 2, 1173.

[13] Yuan, K.; Guo-Wang, P.; Hu, T.; Shi, L.; Zeng, R.; Forster, M.; Pichler, T.; Chen, Y. W.; Scherf, U. Chem. Mater. 2015, 27, 7403.

[14] Budd, P. M.; Msayib, K. J.; Tattershall, C. E.; Ghanem, B. S.; Reynolds, K. J.; Mckeown, N. B.; Fritsch, D. J. Membr. Sci. 2005, 251, 263.

[15] Rose, I.; Bezzu, C. G.; Carta, M.; Comesana-Gandara, B.; Lasseuguette, E.; Ferrari, M. C.; Bernardo, P.; Clarizia, G.; Fuoco, A.; Jansen, J. C.; Hart, K. E.; Liyana-Arachchi, T. P.; Colina, C. M.; Mckeown, N. B. Nat. Mater. 2017, 16, 932.

[16] Ben, T.; Ren, H.; Ma, S. Q.; Cao, D. P.; Lan, J. H.; Jing, X. F.; Wang, W. C.; Xu, J.; Deng, F.; Simmons, J. M.; Qiu, S. L.; Zhu, G. S. Angew. Chem., Int. Ed. 2009, 48, 9457, S9451. 
[17] Ben, T.; Qiu, S. L. CrystEngComm 2013, 15, 17.

[18] Jiang, L. C.; Tian, Y. Y.; Sun, T.; Zhu, Y. L.; Ren, H.; Zou, X. Q.; Ma, Y. H.; Meihaus, K. R.; Long, J. R.; Zhu, G. S. J. Am. Chem. Soc. 2018, 140, 15724.

[19] Hei, Z.; Huang, M.; Luo, Y.; Wang, Y. Polym. Chem. 2016, 7, 770.

[20] Fu, H. X.; Zhang, Z. H.; Fan, W. H.; Wang, S. F.; Liu, Y.; Huang, M. H. J. Mater. Chem. A 2019, 7, 15048.

[21] Chen, Q.; Luo, M.; Hammershoej, P.; Zhou, D.; Han, Y.; Laursen, B. W.; Yan, C. G.; Han, B. H. J. Am. Chem. Soc. 2012, 134, 6084.

[22] Liu, X. X.; Luo, X. S.; Fu, H. X.; Fan, W. H.; Chen, S. L.; Huang, M. H. Chem. Commun. 2020, 56, 2103.

[23] Cote, A. P.; Benin, A. I.; Ockwig, N. W.; O'Keeffe, M.; Matzger, A. J.; Yaghi, O. M. Science 2005, 310, 1166.

[24] Yaghi, O. M.; Li, G. M.; Li, H. L. Nature 1995, 378, 703.

[25] Kandambeth, S.; Dey, K.; Banerjee, R. J. Am. Chem. Soc. 2019, 141, 1807.

[26] Kandambeth, S.; Mallick, A.; Lukose, B.; Mane, M. V.; Heine, T.; Banerjee, R. J. Am. Chem. Soc. 2012, 134, 19524.

[27] Chandra, S.; Kandambeth, S.; Biswal, B. P.; Lukose, B.; Kunjir, S. M.; Chaudhary, M.; Babarao, R.; Heine, T.; Banerjee, R. J. Am. Chem. Soc. 2013, 135, 17853.

[28] Biswal, B. P.; Chandra, S.; Kandambeth, S.; Lukose, B.; Heine, T.; Banerjee, R. J. Am. Chem. Soc. 2013, 135, 5328.

[29] Pachfule, P.; Kandambeth, S.; Diaz, D.; Banerjee, R. Chem. Commun. 2014, 50, 3169.

[30] Biswal, B. P.; Kandambeth, S.; Chandra, S.; Shinde, D. B.; Bera, S.; Karak, S.; Garai, B.; Kharul, U. K.; Banerjee, R. J. Mater. Chem. A 2015, 3, 23664.

[31] Deblase, C. R.; Silberstein, K. E.; Truong, T.; Abruña, H. D.; Dichtel, W. R. J. Am. Chem. Soc. 2013, 135, 16821.

[32] Deblase, C. R.; Hernandez-Burgos, K.; Silberstein, K. E.; Rodriguez-Calero, G. G.; Bisbey, R. P.; Abruna, H. D.; Dichtel, W. R. ACS Nano. 2015, 9, 3178.

[33] Mulzer, C. R.; Shen, L. X.; Bisbey, R. P.; Mckone, J. R.; Zhang, N.; Abruna, H. D.; Dichtel, W. R. ACS Cent. Sci. 2016, 2, 667.

[34] Vitaku, E.; Gannett, C. N.; Carpenter, K. L.; Shen, L. X.; Abruna, H. D.; Dichtel, W. R. J. Am. Chem. Soc. 2020, 142, 16.

[35] Li, Z. P.; Zhi, Y. F.; Feng, X.; Ding, X. S.; Zou, Y. C.; Liu, X. M.; Mu, Y. Chem.-Eur. J. 2015, 21, 12079.

[36] Yang, H.; Wu, H.; Yao, Z. Q.; Shi, B. B.; Xu, Z.; Cheng, X. X.; Pan, F. S.; Liu, G. H.; Jiang, Z. Y.; Cao, X. Z. J. Mater. Chem. A 2018, 6, 583.

[37] Yang, H.; Wu, H.; Xu, Z.; Mu, B. W.; Lin, Z. X.; Cheng, X. X.; Liu, G. H.; Pan, F. S.; Cao, X. Z.; Jiang, Z. Y. J. Membr. Sci. 2018, 561, 79.

[38] Liu, G. H.; Jiang, Z. Y.; Yang, H.; Li, C. D.; Wang, H. J.; Wang, M. D.; Song, Y. M.; Wu, H.; Pan, F. S. J. Membr. Sci. 2019, 572, 557.

[39] Wang, M. D.; Pan, F. S.; Yang, H.; Cao, Y.; Wang, H. J.; Song, Y. M.; Lu, Z.; Sun, M. Z.; Wu, H.; Jiang, Z. Y. J. Mater. Chem. A 2019, 7, 9912 .

[40] Cantillo, D.; Damm, M.; Dallinger, D.; Bauser, M.; Berger, M.; Kappe, C. O. Org. Process Res. Dev. 2014, 18, 1360.

[41] Seo, J. M.; Noh, H.; Jeong, H. Y.; Baek, J. B. J. Am. Chem. Soc. 2019, 141, 11786 .

[42] Mahmood, J.; Lee, E. K.; Jung, M.; Shin, D.; Jeon, I. Y.; Jung, S. M.; Choi, H. J.; Seo, J. M.; Bae, S. Y.; Sohn, S. D.; Park, N.; Oh, J. H.; Shin, H. J.; Baek, J. B. Nat. Commun. 2015, 6, 6486.

[43] Mahmood, J.; Jung, S.; Kim, S.; Park, J.; Yoo, J.; Baek, J. Chem. Mater. 2015, 27, 4860.

[44] Mahmood, J.; Li, F.; Jung, S. M.; Okyay, M. S.; Ahmad, I.; Kim, S. J.; Park, N.; Jeong, H. Y.; Baek, J. B. Nat. Nanotechnol. 2017, 12, 441.
[45] Mahmood, J.; Li, F.; Kim, C.; Choi, H. J.; Gwon, O.; Jung, S. M.; Seo, J. M.; Cho, S. J.; Ju, Y. W.; Jeong, H. Y.; Kim, G.; Baek, J. B. Nano Energy 2018, 44, 304.

[46] Walczak, R.; Kurpil, B.; Savateev, A.; Heil, T.; Schmidt, J.; Qin, Q.; Antonietti, M.; Oschatz, M. Angew. Chem., Int. Ed. 2018, 57, 10765.

[47] Shinde, S. S.; Lee, C. H.; Yu, J.; Kim, D.; Lee, S. U.; Lee, J. ACS Nano. 2018, 12, 596.

[48] Li, M.; Cui, Z.; Pang, S.; Meng, L.; Ma, D.; Li, Y.; Shi, Z.; Feng, S. J. Mater. Chem. C 2019, 7, 11919.

[49] Ma, J. X.; Li, J.; Chen, Y. F.; Ning, R.; Ao, Y. F.; Liu, J. M.; Sun, J. L.; Wang, D. X.; Wang, Q. Q. J. Am. Chem. Soc. 2019, 141, 3843.

[50] Yang, Y.; He, X. Y.; Zhang, P. H.; Andaloussi, Y. H.; Zhang, H. L.; Jiang, Z. Y.; Chen, Y.; Ma, S. Q.; Cheng, P.; Zhang, Z. J. Angew. Chem., Int. Ed. 2020, 59, 3678.

[51] Abrahams, B. F.; Egan, S. J., Robson, R. J. Am. Chem. Soc. 1999, 121,3535 .

[52] Hurd, J. A.; Vaidhyanathan, R.; Thangadurai, V.; Ratcliffe, C. I.; Moudrakovski, I. L.; Shimizu, G. K. H. Nat. Chem. 2009, 1, 705.

[53] Kim, S. R.; Dawson, K. W.; Gelfand, B. S.; Taylor, J. M.; Shimizu, G. K. H. J. Am. Chem. Soc. 2013, 135, 963.

[54] Yang, R.; Li, L.; Xiong, Y.; Li, J.; Zhou, H. C.; Su, C. Y. Chem.Asian J.2010, 5, 2358.

[55] Zhao, N. N.; Li W. J.; Sun, C. Y.; Bian, Y. Z.; Wang, H. L.; Chang, Z. D.; Fan, H. X. Solid State Sci. 2012, 14, 317.

[56] Chaudhari, A. K.; Nagarkar, S. S.; Joarder, B.; Ghosh, S. K. Cryst. Growth Des. 2013, 13, 3716.

[57] Rimoldi, M.; Nakamura, A.; Vermeulen, N. A.; Henkelis, J. J.; Blackburn, A. K.; Hupp, J. T.; Stoddart, J. F.; Farha, O. K. Chem. Sci. 2016, 7, 4980.

[58] Hong, S.; Rohman, M. R.; Jia, J. T.; Kim, Y.; Moon, D.; Kim, Y.; Ko, Y. H.; Lee, E.; Kim, K. Angew. Chem., Int. Ed. 2015, 54, 13241.

[59] Chen, Q.; Luo, M.; Hammershoej, P.; Zhou, D.; Han, Y.; Laursen, B. W.; Yan, C. G.; Han, B. H. J. Am. Chem. Soc. 2012, 134, 6084.

[60] Zhou, J. X.; Luo, X. S.; Liu, X. X.; Qiao, Y.; Wang, P. F.; Mecerreyes, D.; Bogliotti, N.; Chen, S. L.; Huang, M. H. J. Mater. Chem. A 2018, 6, 5608 .

[61] Liu, X. X.; Luo, X. S.; Deng, H. L.; Fan, W. H.; Wang, S. F.; Yang, C. J.; Sun, X. Y.; Chen, S. L.; Huang, M. H. Chem. Mater. 2019, 31,5421 .

[62] Yu, H.; Shen, C. J.; Wang, Z. G. ChemPlusChem 2013, 78, 498.

[63] Karmakar, A.; Kumar, A.; Chaudhari, A. K.; Samanta, P.; Desai, A. V.; Krishna, R.; Ghosh, S. K. Chem.-Eur. J. 2016, 22, 4931.

[64] Wang, L.; Jia, J. T.; Faheem, M.; Tian, Y. Y.; Zhu, G. S. J. Ind. Eng. Chem. 2018, 67, 373.

[65] Ullah, R.; Atilhan, M.; Anaya, B.; Al-Muhtaseb, S.; Aparicio, S.; Patel, H.; Thirion, D.; Yavuz, C. T. ACS Appl. Mater. Interfaces 2016, 8, 20772.

[66] Kejik, M.; Moravec, Z.; Barnes, C. E.; Pinkas, J. Microporous Mesoporous Mater. 2017, 240, 205.

[67] Xu, S. J.; He, J.; Jin, S. B.; Tan, B. E. J. Colloid Interface Sci. 2018, $509,457$.

[68] Zhang, M. C.; Li, Y.; Bai, C. Y.; Guo, X. H.; Han, J.; Hu, S.; Jiang, H. Q.; Tan, W.; Li, S. J.; Ma, L. J. ACS Appl. Mater. Interfaces 2018, 10, 28936.

[69] Katsoulidis, A. P.; Kanatzidis, M. G. Chem. Mater. 2011, 23, 1818.

[70] Katsoulidis, A. P.; Kanatzidis, M. G. Chem. Mater. 2012, 24, 471.

[71] Ding, M. L.; Jiang, H. L. Chem. Commun. 2016, 52, 12294.

[72] Pareek, K.; Rohan, R.; Chen, Z.; Zhao, D.; Cheng, H. S. Int. J. Hydrogen Energy 2017, 42, 6801.

[73] Kang, D. W.; Lim, K. S.; Lee, K. J.; Lee, J. H.; Lee, W. R.; Song, J. 
H.; Yeom, K. H.; Kim, J. Y.; Hong, C. S. Angew. Chem., Int. Ed. 2016, $55,16123$.

[74] Kang, D. W.; Song, J. H.; Lee, K. J.; Lee, H. G.; Kim, J. E.; Lee, H. Y.; Kim, J. Y.; Hong, C. S. J. Mater. Chem. A 2017, 5, 17492.

[75] Kang, D. W.; Lee, K. A.; Kang, M.; Kim, J. M.; Moon, M.; Choe, J. H.; Kim, H.; Kim, D. W.; Kim, J. Y.; Hong, C. S. J. Mater. Chem. A 2020, $8,1147$.

[76] Chen, T. T.; Tan, H. L.; Chen, Q. B.; Gu, L. N..; Wei, Z. S.; Liu, H. L. ACS Appl. Mater. Interfaces 2019, 11, 48402.

[77] Ji, G. P.; Yang, Z. Z.; Zhang, H. Y.; Zhao, Y. F.; Yu, B.; Ma, Z. S.; Liu, Z. M. Angew. Chem., Int. Ed. 2016, 55, 9685.

[78] Huang, L.; He, M.; Chen, B.; Cheng, Q.; Hu, B. ACS Sustainable Chem. Eng. 2017, 5, 4050

[79] Bhat, S. A.; Das, C.; Maji, T. K. J. Mater. Chem. A 2018, 6, 19834.

[80] Liu, M.; Yao, C.; Liu, C.; Xu, Y. Sci. Rep. 2018, 8, 14072.

[81] Bera, R.; Ansari, M.; Alam, A.; Das, N. ACS Appl. Polym. Mater. 2019, 1, 959.

[82] Vinodh, R.; Abidov, A.; Peng, M. M.; Babu, C. M.; Palanichamy, M.; Cha, W. S.; Jang, H. J. Ind. Eng. Chem. 2015, 32, 273.

[83] Jiang, K.; Zhao, H. R.; Dai, J. X.; Kuang, D.; Fei, T.; Zhang, T. ACS
Appl. Mater. Interfaces 2016, 8, 25529.

[84] Ma, H. P.; Ren, H.; Zou, X. Q; Sun, F. X.; Yan, Z. J.; Cai, K.; Wang, D. Y.; Zhu, G. S. J. Mater. Chem. A 2013, 1, 752.

[85] Yuan, R. R.; Ren, H.; Yan, Z. J.; Wang, A. F.; Zhu, G. S. Polym. Chem. 2014, 5, 2266.

[86] Shen, X. S.; Faheem, M.; Matsuo, Y.; Aziz, S.; Zhang, X.; Li, Y. H.; Song, J.; Tian, Y. Y.; Zhu, G. S. J. Mater. Chem. A 2019, 7, 2507.

[87] Patra, B. C.; Khilari, S.; Manna, R. N.; Mondal, S.; Pradhan, D.; Pradhan, A.; Bhaumik, A. ACS Catal. 2017, 7, 6120.

[88] Bhanja, P.; Das, S. K.; Bhunia, K.; Pradhan, D.; Hayashi, T.; Hijikata, Y.; Irle, S.; Bhaumik, A. ACS Sustainable Chem. Eng. 2017, 6, 202.

[89] Das, S. K.; Bhanja, P.; Kundu, S. K.; Mondal, S.; Bhaumik, A. ACS Appl. Mater. Interfaces 2018, 10, 23813.

[90] Kochergin, Y. S.; Schwarz, D.; Acharjya, A.; Ichangi, A.; Kulkarni, R.; Eliášová, P.; Vacek, J.; Schmidt, J.; Thomas, A.; Bojdys, M. J. Angew. Chem., Int. Ed. 2018, 57, 14188.

[91] Kochergin, Y. S.; Noda, Y.; Kulkarni, R.; Akodáková, K.; Tarábek, J.; Schmidt, J.; Bojdys, M. J. Macromolecules 2019, 52, 7696. 